\title{
Coupling Non-Linear Stokes and Darcy Flow using Mortar Finite Elements
}

\author{
V.J. Ervin * $\quad$ E.W. Jenkins * S. Sun * \\ Department of Mathematical Sciences \\ Clemson University, Clemson, SC, 29634-0975, USA.
}

\begin{abstract}
We study a system composed of a non-linear Stokes flow in one subdomain coupled with a nonlinear porous medium flow in another subdomain. Special attention is paid to the mathematical consequence of the shear-dependent fluid viscosity for the Stokes flow and the velocity-dependent effective viscosity for the Darcy flow. Motivated by the physical setting, we consider the case where only flow rates are specified on the inflow and outflow boundaries in both subdomains. We recast the coupled Stokes-Darcy system as a reduced matching problem on the interface using a mortar space approach. We prove a number of properties of the nonlinear interface operator associated with the reduced problem, which directly yield the existence, uniqueness and regularity of a variational solution to the system. We further propose and analyze a numerical algorithm based on mortar finite elements for the interface problem and conforming finite elements for the subdomain problems. Optimal a priori error estimates are established for the interface and subdomain problems, and a number of compatibility conditions for the finite element spaces used are discussed. Numerical simulations are presented to illustrate the algorithm and to compare two treatments of the defective boundary conditions.
\end{abstract}

Key words. Generalized non-linear Stokes flow; coupled Stokes and Darcy flow; defective boundary condition

AMS Mathematics subject classifications. 65N30

\section{Introduction}

The problem of approximating coupled Stokes-Darcy flow has received considerable attention in the mathematics community over the past ten years. Many of the applications considered use a Newtonian fluid in both the Stokes and Darcy regions, where the motivating problem is often a coupled surface water / groundwater model (see, for instance, [6, 14, 18, 22, 24, 27, 28]). Our interest in coupled flows arises from filtration applications, which continues to be an active area of research (see, for instance, $[3,12,20,26,23]$ ). The purpose of the filtration mechanism can be the removal of particulates $[23,3,26,29]$ or impurities [20], but in all cases the availability of an accurate and efficient simulation tool would aid in the design and assessment of effective filtration devices.

*email: vjervin@clemson.edu, lea@clemson.edu, shuyu@clemson.edu. 
Our particular focus is on the effective removal of debris particles from a molten polymer $[29,10,5]$, which is a non-Newtonian fluid. Earlier work on this filtration problem focused only on the flow in the porous, or Darcy, region [1,21, 25, 29, 30], but analysis of the fully coupled problem is essential to accurately simulate the transport of the suspended particles into the filter. The fully coupled problem for non-Newtonian Stokes and Darcy flows was initially analyzed in [11]. Motivated by numerical implementation considerations, we have recast this coupled problem as a matching problem on the interface between the domains. This approach naturally gives rise to a parallel algorithm for the subproblems in the Stokes domain $\Omega_{f}$ and the Darcy domain $\Omega_{p}$, and it also allows one to combine existing codes for Stokes and Darcy simulations to solve the coupled problem. Key ideas from $[7,22,14,17]$ are used in the new formulation.

In the literature, two basic approaches have been used to analyze the coupled problem considered herein: Stokes-Darcy coupling and Stokes-Laplace coupling. In Stokes-Darcy coupling, the velocity and pressure are resolved in both the fluid flow and the porous media domains. For the StokesLaplace formulation the velocity and pressure are the unknowns in the fluid flow domain and the pressure is the only unknown in the porous media domain.

In [7] Discacciati and Quateroni studied the coupled Stokes-Laplace formulation. After showing existence and uniqueness for the coupled problem they reformulated the problem, using the Steklov-Poincaré operator, as an interface problem for the interfacial pressure. Parallel and serial implementations for the numerical approximation of the interface problem were reported in $[8,9]$. There has been considerable work done on efficient numerical solution algorithms for the Stokes-Laplace formulation. We refer the reader to $[4,19,24]$ and the references therein.

Using a Lagrange multiplier technique Layton, Schieweck, and Yotov [22] (see [28] also) introduced and studied the Stokes-Darcy coupling approach. Galvis and Sarkis in [14] extended this approach and showed how the Lagrange multiplier space could be more appropriately defined. In [13] they investigated efficient preconditioning strategies for the Stokes-Darcy formulation.

Our interest herein is the Stokes-Darcy coupling approach. Denoting by $\mathbf{u}_{f}, p_{f}, \mathbf{u}_{p}, p_{p}$ the velocity and pressure in the fluid flow domain $\Omega_{f}$ and the porous media domain $\Omega_{p}$, respectively, we have the following boundary conditions holding along the interface $\Gamma$ :

$$
\begin{aligned}
& \mathbf{u}_{f} \cdot \mathbf{n}_{f}+\mathbf{u}_{p} \cdot \mathbf{n}_{p}=0, \quad \text { (Conservation of mass) } \\
& p_{f}-\left(\boldsymbol{\sigma}_{f} \mathbf{n}_{f}\right) \cdot \mathbf{n}_{f}=p_{p}, \quad \text { (Balance of interfacial pressure) }
\end{aligned}
$$

and a boundary condition for the tangential stress in $\Omega_{f}$ on $\Gamma$. (The boundary condition for the tangential stress becomes a natural boundary condition for the variational formulation on $\Omega_{f}$.) In (1.2) $\boldsymbol{\sigma}_{f}$ denotes the extra stress tensor in $\Omega_{f}$. For the coupled problem under consideration, fully described in Section 2 below, given an interfacial pressure $p_{I}$, the Stokes and Darcy problems are independently solvable in $\Omega_{f}$ and $\Omega_{p}$, respectively, to yield $\mathbf{u}_{f}^{*}\left(p_{I}\right), p_{f}^{*}\left(p_{I}\right), \mathbf{u}_{p}^{*}\left(p_{I}\right), p_{p}^{*}\left(p_{I}\right)$. Thus, we investigate the problem of determining $\lambda$ such that

$$
\Lambda(\lambda):=\mathbf{u}_{f}^{*}(\lambda) \cdot \mathbf{n}_{f}+\mathbf{u}_{p}^{*}(\lambda) \cdot \mathbf{n}_{p}=0 .
$$

The matching problem lies on the interface $\Gamma$. For the discrete approximation we introduce a "mortar space" for the representation of the interfacial pressure of $\Gamma[17,16]$. The mortar space discretization is independent of the partitions of $\Omega_{f}$ and $\Omega_{p}$. However a compatibility condition for the mortar space discretization and the partitions of $\Omega_{f}$ and $\Omega_{p}$ is required (see Assumptions 4.4, 4.5). 
For the modeling equations describing the fluid flow we consider the case of a shear thinning fluid, where the fluid's viscosity is a nonlinear function of the magnitude of the deformation tensor. Additionally we assume that only flow rates are specified for the inflow and outflow boundaries (i.e. defective boundary conditions).

The paper is organized as follows. In Section 2 we formally describe the modeling equations and assumptions on the non-linear functions modeling the fluid's viscosity. Variational formulations for the Stokes problem in $\Omega_{f}$, and Darcy problem in $\Omega_{p}$, are given in Section 3 where the interfacial pressure, $p_{I}$, is treated as a known quantity for both problems. Existence and uniqueness of the problems is verified and solutions are shown to depend continuously on $p_{I}$. The matching problem of determining $p_{I}$ such that (1.1) is satisfied is then formulated and shown to have a unique solution. In Section 4 we present the finite element approximation scheme and analysis. The analysis for the discrete problem follows the same pattern as the continuous problem. First the discrete Stokes and Darcy problems are separately considered. Existence and uniqueness of the discrete approximations is verified and error estimates between the continuous solutions and discrete approximations are derived. The discrete matching problem is then formulated and shown to have a unique solution. A combined error estimate is then given for the solution between the coupled Stokes-Darcy problem and its discrete approximation. In Section 5 a numerical example is given for two different treatments of the defective boundary conditions.

\section{$2 \quad$ Modeling Equations}

For convenience, we work with Hilbert spaces instead of general Sobolev spaces for our weak formulations. This requires us to assume that the viscosity is bounded from above. A general formulation involving the spaces $W^{1, r}\left(\Omega_{f}\right)$ and $W^{1 / r, r^{\prime}}(\Gamma)$ can be found in [11]. We would like to mention that there exists a certain physical limit for the viscosity, so our assumption is actually physically reasonable. The power law, for example, is an approximation of the reality, and the implication from the power law that the viscosity goes to infinity when the deformation goes to zero is not physically correct as the power law does not apply to the case of a zero strain tensor.

For convenience of implementation, we use Einstein's notation in addition to the vector notation. All repeated subscript indices imply summation over all dimensions except the subscript $m$ for the tangential vector $t_{m i}$, where we list the summation of $m$ explicitly.

Let $\Omega \subset \mathbb{R}^{n}, n=2$ or 3 , denote the flow domain of interest. Let $\Omega_{f} \subset \Omega$ and $\Omega_{p} \subset \Omega$ be bounded Lipschitz domains for the non-linear Stokes flow and non-linear Darcy flow, respectively. In this work, we consider only two subdomains, and assume $\Omega=\Omega_{f} \cup \Omega_{p} \cup \Gamma_{I}$, where $\Gamma_{I}:=\partial \Omega_{f} \cap \partial \Omega_{p}$, denoting a smooth interface between the subdomains. 
We first consider the non-linear Stokes flow in $\Omega_{f}$.

$$
\begin{aligned}
\frac{\partial p}{\partial x_{i}}-\frac{\partial \boldsymbol{\sigma}_{i j}}{\partial x_{j}} & =f_{i}, \quad \text { in } \Omega_{f}, \\
\frac{\partial u_{i}}{\partial x_{i}} & =0, \quad \text { in } \Omega_{f}, \\
\boldsymbol{\sigma}_{i j} & =2 \nu_{f}(|D \mathbf{u}|)(D \mathbf{u})_{i j}, \quad \text { in } \Omega_{f}, \\
u_{i} & =0, \quad \text { on } \Gamma_{f, D}, \\
\left(\alpha_{S} u_{i}+\boldsymbol{\sigma}_{i j} n_{j}\right) t_{m i} & =0, \quad \text { on } \Gamma_{I}, m=1, \cdots, d-1, \\
\int_{\Gamma_{f, k}} u_{i} n_{i} d s & =\int_{\Gamma_{f, k}} q_{f, k} d s=q_{f, k} \operatorname{meas}\left(\Gamma_{f, k}\right), \quad k=1,2, \cdots, K_{f} .
\end{aligned}
$$

Note that $\mathbf{u}_{B}$ and $\boldsymbol{\tau}_{B}$ denote vector functions on the domain boundary, where $\partial \Omega_{f}=\Gamma_{f, D} \cup \Gamma_{f, N} \cup$ $\Gamma_{f, F} \cup \Gamma_{I}$ (this is understood as $\partial \Omega_{f}=\overline{\Gamma_{f, D} \cup \Gamma_{f, N} \cup \Gamma_{f, F} \cup \Gamma_{I}}$ and similar notational simplification applied to the rest of this paper), $\Gamma_{f, F}:=\bigcup_{k=1}^{K_{f}} \Gamma_{f, k}$ and $K_{f}$ is the number of defective boundaries in the Stokes flow domain. The boundaries $\Gamma_{f, D}, \Gamma_{f, N}, \Gamma_{f, F}$, and $\Gamma_{I}$ are pairwise disjoint, and their definitions are clear from the following modeling equations for Stokes flow. We use $D$ to denote the symmetric gradient operator. In other words, $D \mathbf{u}$ is the deformation tensor $(D \mathbf{u})_{i j}=\frac{1}{2}\left(\frac{\partial u_{i}}{\partial x_{j}}+\frac{\partial u_{j}}{\partial x_{i}}\right)$. The viscosity $\nu_{f}$ depends nonlinearly on $|D \mathbf{u}|$. We assume that $\nu_{f}$ is bounded from above and from below; that is, there exist $\nu_{\min }>0$ and $\nu_{\max }$ such that $\nu_{\min } \leq \nu_{f}(|D \mathbf{u}|) \leq \nu_{\max }$ for all $\mathbf{u}$. We assume that meas $\left(\Gamma_{f, N}\right)>0$ and meas $\left(\Gamma_{I}\right)>0$. On $\Gamma_{I}$, we impose the Beavers-Joseph-Saffman (BJS) condition (2.9). On each $\Gamma_{f, k}$, we impose a defective boundary condition, where the averaged flow rate per area is imposed; or equivalently, the integral of the flow rate $q_{f, k}$ is specified on $\Gamma_{f, k}$. Note that we assume that $\alpha_{S}$ is a scalar for simplicity of presentation, but all results in this paper can be easily extended to treat a full tensor $\alpha_{S, i j}$.

The Darcy flow in $\Omega_{p}$ is modeled using the equations:

$$
\begin{aligned}
\nu_{p}(|\mathbf{u}|) R_{i j} u_{j}+\frac{\partial p}{\partial x_{i}} & =f_{i}, \quad \text { in } \Omega_{p}, \\
\frac{\partial u_{i}}{\partial x_{i}} & =0, \quad \text { in } \Omega_{p}, \\
u_{i} n_{i} & =0, \quad \text { on } \Gamma_{p, N}, \\
p & =p_{B}, \quad \text { on } \Gamma_{p, D}, \\
\int_{\Gamma_{p, k}} u_{i} n_{i} d s & =\int_{\Gamma_{p, k}} q_{p, k} d s=q_{p, k} \operatorname{meas}\left(\Gamma_{p, k}\right), \quad k=1,2, \cdots, K_{p},
\end{aligned}
$$

where $\partial \Omega_{p}=\Gamma_{p, N} \cup \Gamma_{p, D} \cup \Gamma_{p, F} \cup \Gamma_{I}$. We assume that the permeability tensor $\mathbf{K}$ associated with the porous medium is symmetric positive definite, and we denote its inverse by the flow resistivity tensor $\mathbf{R}$; that is, $\mathbf{R}=\mathbf{K}^{-1}$. We also assume that $\nu_{p}(|\mathbf{u}|)$ is bounded from above by $\nu_{\max }$ and from below by $\nu_{\min }>0$. Similar to the Stokes region, we have imposed a defective boundary condition on the Darcy region boundary $\Gamma_{p, k}$, with $q_{p, k} \in \mathbb{R}$ being the imposed averaged flow rate; $q_{p} \in \mathbb{R}^{K_{p}}$ may be also viewed as a piecewise constant function defined on $\Gamma_{p, F}=\bigcup_{k=1}^{K_{p}} \Gamma_{p, k}$. 
To couple the two flow systems, we impose conservation of mass and balance of the normal forces across the interface $\Gamma_{I}$ :

$$
\begin{array}{r}
{\left.\left[u_{i} n_{i}\right]\right|_{\Gamma_{I}}=0,} \\
{\left.\left[p-\sigma_{i j} n_{i} n_{j}\right]\right|_{\Gamma_{I}}=0,}
\end{array}
$$

where we extend $\boldsymbol{\sigma}_{i j}$ by zero to $\Omega_{p}$ for notational ease; that is $\left.\boldsymbol{\sigma}_{i j}\right|_{\Omega_{p}}=0$. We will use $p_{f}:=\left.p\right|_{\Omega_{f}}$, $\mathbf{u}_{f}:=\left.\mathbf{u}\right|_{\Omega_{f}}, \mathbf{f}_{f}:=\left.\mathbf{f}\right|_{\Omega_{f}}, p_{p}:=\left.p\right|_{\Omega_{p}}, \mathbf{u}_{p}:=\left.\mathbf{u}\right|_{\Omega_{p}}, \mathbf{f}_{p}:=\left.\mathbf{f}\right|_{\Omega_{p}}$ to emphasize the corresponding variables applied to a specific region, although we will simply write $p$, $\mathbf{u}$ and $\mathbf{f}$ when the region we refer to is clear from the context. On the interface $\Gamma_{I}, \mathbf{n}_{f}=\mathbf{n}_{\partial \Omega_{f}}=-\mathbf{n}_{p}=-\mathbf{n}_{\partial \Omega_{p}}$ denotes the unit normal vector pointing from $\Omega_{f}$ toward $\Omega_{p}$. When we restrict our attention to the Stokes (or Darcy) region, we simply write $\mathbf{n}_{f}$ (or $\mathbf{n}_{p}$ ) as $\mathbf{n}$.

Throughout the paper, we use $C$ to denote a generic positive constant, and $\epsilon$ to denote a fixed positive constant that may be chosen arbitrarily small. We assume that the nonlinear functions $\nu_{f}(|D \mathbf{u}|) D \mathbf{u}$ and $\nu_{p}(|\mathbf{u}|) \mathbf{u}$ are uniformly continuous with regard to $D \mathbf{u}$ and $\mathbf{u}$, respectively; that is, there exists a constant $C>0$ such that

$$
\begin{array}{cc}
\left|\nu_{f}(|\mathbf{T}|) \mathbf{T}-\nu_{f}(|\mathbf{S}|) \mathbf{S}\right| \leq C|\mathbf{T}-\mathbf{S}|, \quad \forall \mathbf{T} \in \mathbb{R}^{d \times d}, \quad \forall \mathbf{S} \in \mathbb{R}^{d \times d}, \\
\left|\nu_{p}(|\mathbf{u}|) \mathbf{u}-\nu_{p}(|\mathbf{v}|) \mathbf{v}\right| \leq C|\mathbf{u}-\mathbf{v}|, \quad \forall \mathbf{u} \in \mathbb{R}^{d}, \quad \forall \mathbf{v} \in \mathbb{R}^{d} .
\end{array}
$$

In addition, we assume that $\nu_{f}(|\cdot|)$ and $\nu_{p}(|\cdot|)$ are strictly monotone; namely, there exists a constant $C>0$ such that:

$$
\begin{gathered}
\left(\nu_{f}(|\mathbf{T}|) \mathbf{T}-\nu_{f}(|\mathbf{S}|) \mathbf{S}\right):(\mathbf{T}-\mathbf{S}) \geq C(\mathbf{T}-\mathbf{S}):(\mathbf{T}-\mathbf{S}), \quad \forall \mathbf{T} \in \mathbb{R}^{d \times d}, \forall \mathbf{S} \in \mathbb{R}^{d \times d}, \\
\left(\nu_{p}(|\mathbf{u}|) \mathbf{u}-\nu_{p}(|\mathbf{v}|) \mathbf{v}\right) \cdot(\mathbf{u}-\mathbf{v}) \geq C(\mathbf{u}-\mathbf{v}) \cdot(\mathbf{u}-\mathbf{v}), \quad \forall \mathbf{u} \in \mathbb{R}^{d}, \forall \mathbf{v} \in \mathbb{R}^{d} .
\end{gathered}
$$

\section{Variational Formulations with Mortar Spaces}

We first consider the Stokes flow and the Darcy flow problems separately as if they were two independent processes. This corresponds to the modularity of their code implementation, where it is desirable to implement and test individual codes for separate Stokes flow and Darcy flow before numerically coupling them.

The variational formulations for the fully coupled problem is presented and analyzed in [11].

\subsection{Stokes flow}

We now restrict our attention to the Stokes flow problem, by assuming the interface pressure $p_{I}$ is given. The equations to be solved include (2.4)-(2.10) together with the balance of the normal forces across the interface $\Gamma_{I}$ :

$$
\begin{gathered}
\boldsymbol{\sigma}_{i j} n_{i} n_{j}-p=-p_{I}, \quad \text { on } \Gamma_{I} \\
\text { Let } \quad \mathbf{X}_{f}=\left\{\mathbf{v}: \mathbf{v} \in\left(H^{1}\left(\Omega_{f}\right)\right)^{d},\left.\mathbf{v}\right|_{\Gamma_{f, D}}=\mathbf{0}\right\}
\end{gathered}
$$


To derive the weak formulation, we multiply (2.4) by a vector function $\mathbf{v} \in \mathbf{X}_{f}$, integrate over $\Omega_{f}$ and apply the divergence theorem (Green's theorem) to obtain

$$
\begin{aligned}
\left(f_{i}, v_{i}\right)_{\Omega_{f}}= & \int_{\Omega_{f}}\left(\frac{\partial p}{\partial x_{i}}-\frac{\partial \boldsymbol{\sigma}_{i j}}{\partial x_{j}}\right) v_{i} d x \\
= & \int_{\Omega_{f}}\left(\boldsymbol{\sigma}_{i j}(D \mathbf{v})_{i j}-p \frac{\partial v_{i}}{\partial x_{i}}\right) d x-\int_{\partial \Omega_{f}}\left(\boldsymbol{\sigma}_{i j} n_{j}-p n_{i}\right) v_{i} d s \\
= & \int_{\Omega_{f}}\left(2 \nu_{f}(|D \mathbf{u}|)(D \mathbf{u})_{i j}(D \mathbf{v})_{i j}-p \frac{\partial v_{i}}{\partial x_{i}}\right) d x-\int_{\Gamma_{f, N}} \boldsymbol{\tau}_{B, i} v_{i} d s \\
& \quad-\int_{\Gamma_{I}}\left(\boldsymbol{\sigma}_{i j} n_{j}-p n_{i}\right) v_{i} d s-\int_{\Gamma_{f, F}}\left(\boldsymbol{\sigma}_{i j} n_{j}-p n_{i}\right) v_{i} d s
\end{aligned}
$$

Noting that

$$
\sum_{m=1}^{d-1} t_{m i} t_{m j}+n_{i} n_{j}=\delta_{i j}
$$

we can treat the BJS slip boundary condition as

$$
\begin{aligned}
-\int_{\Gamma_{I}}\left(\boldsymbol{\sigma}_{i j} n_{j}-p n_{i}\right) v_{i} d s & =-\int_{\Gamma_{I}}\left(\boldsymbol{\sigma}_{i j} n_{j}-p n_{i}\right) \delta_{i k} v_{k} d s \\
& =-\sum_{m=1}^{d-1} \int_{\Gamma_{I}}\left(\boldsymbol{\sigma}_{i j} n_{j}-p n_{i}\right) t_{m i} t_{m k} v_{k} d s-\int_{\Gamma_{I}}\left(\boldsymbol{\sigma}_{i j} n_{j}-p n_{i}\right) n_{i} n_{k} v_{k} d s \\
& =-\sum_{m=1}^{d-1} \int_{\Gamma_{I}}\left(\boldsymbol{\sigma}_{i j} n_{j} t_{m i}\right)\left(v_{k} t_{m k}\right) d s-\int_{\Gamma_{I}}\left(\boldsymbol{\sigma}_{i j} n_{i} n_{j}-p\right)\left(n_{k} v_{k}\right) d s \\
& =\sum_{m=1}^{d-1} \int_{\Gamma_{I}} \alpha_{S}\left(u_{i} t_{m i}\right)\left(v_{k} t_{m k}\right) d s+\int_{\Gamma_{I}} p_{I} n_{k} v_{k} d s .
\end{aligned}
$$

As for the defective boundary conditions on $\Gamma_{f, F}$, a total flow rate is specified on each of its pieces $\Gamma_{f, k}$, but the traction vector on the boundary is not imposed. We follow Ervin et al.'s approach [11] and use a Lagrange multiplier method for treating the defective boundary conditions on $\Gamma_{f, F}$ (which for a sufficiently smooth function is equivalent to the condition that the traction vector is a constant vector normal to the boundary surface). Thus the traction integral over $\Gamma_{f, F}$ is replaced by

$$
-\int_{\Gamma_{f, F}}\left(\boldsymbol{\sigma}_{i j} n_{j}-p n_{i}\right) v_{i} d s \longrightarrow-\sum_{k=1}^{K_{f}} \beta_{f, k} \int_{\Gamma_{f, k}} v_{i} n_{i} d s=-\int_{\Gamma_{f, F}} \beta_{f} v_{i} n_{i} d s,
$$

where in the last equality the Lagrange multiplier $\beta_{f} \in \mathbb{R}^{K_{f}}$ is to be viewed as a piecewise constant function on $\Gamma_{f, F}$, with $\beta_{f} n_{i}$ representing the traction vector on the boundary. 
Combining the above results, we have

$$
\begin{aligned}
\left(f_{i}, v_{i}\right)_{\Omega_{f}} & =\int_{\Omega_{f}} 2 \nu_{f}(|D \mathbf{u}|)(D \mathbf{u})_{i j}(D \mathbf{v})_{i j} d x-\int_{\Omega_{f}} p \frac{\partial v_{i}}{\partial x_{i}} d x \\
& -\int_{\Gamma_{f, N}} \boldsymbol{\tau}_{B, i} v_{i} d s-\int_{\Gamma_{f, F}} \beta_{f} v_{i} n_{i} d s \\
& +\sum_{m=1}^{d-1} \int_{\Gamma_{I}} \alpha_{S}\left(u_{i} t_{m i}\right)\left(v_{k} t_{m k}\right) d s+\int_{\Gamma_{I}} p_{I} v_{i} n_{i} d s .
\end{aligned}
$$

The averaged flow rates are imposed by

$$
\int_{\Gamma_{f, F}} \gamma_{f} u_{i} n_{i} d s=\sum_{k=1}^{K_{f}} \int_{\Gamma_{f, k}} \gamma_{f, k} q_{f, k} d s=\sum_{k=1}^{K_{f}} \gamma_{f, k} q_{f, k} \operatorname{meas}\left(\Gamma_{f, k}\right),
$$

where $\gamma_{f}$ is a piecewise constant function on $\Gamma_{f, F}$. The divergence-free equation (2.5) is weakly imposed using

$$
\int_{\Omega_{f}} \frac{\partial u_{i}}{\partial x_{i}} q=0, \quad \forall q \in L^{2}\left(\Omega_{f}\right)
$$

We now introduce

$$
\begin{aligned}
a_{f}(\mathbf{u}, \mathbf{v}) & :=\int_{\Omega_{f}} 2 \nu_{f}(|D \mathbf{u}|)(D \mathbf{u})_{i j}(D \mathbf{v})_{i j} d x+\sum_{m=1}^{d-1} \int_{\Gamma_{I}} \alpha_{S}\left(u_{i} t_{m i}\right)\left(v_{k} t_{m k}\right) d s, \\
b_{f}(\mathbf{v}, p) & :=\int_{\Omega_{f}} \frac{\partial v_{i}}{\partial x_{i}} p d x, \\
b_{f, B}\left(\mathbf{v}, \beta_{f}\right) & :=\int_{\Gamma_{f, F}} \beta_{f} v_{i} n_{i} d s=\sum_{k=1}^{K_{f}} \beta_{f, k} \int_{\Gamma_{f, k}} v_{i} n_{i} d s \\
l_{f}(\mathbf{v}) & :=\left(f_{i}, v_{i}\right)_{\Omega_{f}}+\left\langle\boldsymbol{\tau}_{B}, \mathbf{v}\right\rangle_{\Gamma_{f, N}}-\left\langle p_{I}, \mathbf{v} \cdot \mathbf{n}\right\rangle_{\Gamma_{I}},
\end{aligned}
$$

where $\left\langle\boldsymbol{\tau}_{B}, \mathbf{v}\right\rangle_{\Gamma_{f, N}}$ denotes a duality pairing between $\left(H^{-\frac{1}{2}}\left(\Gamma_{f, N}\right)\right)^{d}$ and $\left(H^{\frac{1}{2}}\left(\Gamma_{f, N}\right)\right)^{d}$, and $\left\langle p_{I}, \mathbf{v} \cdot \mathbf{n}\right\rangle_{\Gamma_{I}}$ a duality pairing between $H^{-\frac{1}{2}}\left(\Gamma_{I}\right)$ and $H^{\frac{1}{2}}\left(\Gamma_{I}\right)$.

The weak formulation for the Stokes flow is: Given $\mathbf{f} \in \mathbf{X}_{f}^{*}, \boldsymbol{\tau}_{B} \in\left(H^{-\frac{1}{2}}\left(\Gamma_{f, N}\right)\right)^{d}, q_{f} \in \mathbb{R}^{K_{f}}$, and $p_{I} \in H^{-\frac{1}{2}}\left(\Gamma_{I}\right)$, determine $\left(\mathbf{u}, p, \beta_{f}\right) \in \mathbf{X}_{f} \times L^{2}\left(\Omega_{f}\right) \times \mathbb{R}^{K_{f}}$ such that

$$
\begin{aligned}
a_{f}(\mathbf{u}, \mathbf{v})-b_{f}(\mathbf{v}, p)-b_{f, B}\left(\mathbf{v}, \beta_{f}\right) & =l_{f}(\mathbf{v}), \quad \forall \mathbf{v} \in \mathbf{X}_{f}, \\
b_{f}(\mathbf{u}, q)+b_{f, B}\left(\mathbf{u}, \gamma_{f}\right) & =b_{f, B}\left(q_{f} \mathbf{n}_{f}, \gamma_{f}\right), \quad \forall\left(q, \gamma_{f}\right) \in L^{2}\left(\Omega_{f}\right) \times \mathbb{R}^{K_{f}} .
\end{aligned}
$$

Again, as remarked above, the term $q_{f} \mathbf{n}_{f}$ is to be interpreted for $q_{f}$ a piecewise constant function on $\Gamma_{f, N}$. 
We first establish an inf-sup condition before presenting our theorem on the existence, uniqueness and regularity of a solution. Let

$$
\begin{aligned}
\mathbf{Z}_{f, B} & :=\left\{\mathbf{v} \in \mathbf{X}_{f}: b_{f, B}\left(\mathbf{v}, \beta_{f}\right)=0, \forall \beta_{f} \in \mathbb{R}^{K_{f}}\right\}, \\
\mathbf{Z}_{f, 0} & :=\left\{\mathbf{v} \in \mathbf{X}_{f}:\left.\mathbf{v}\right|_{\Gamma_{f, F}}=\mathbf{0}\right\}, \\
\mathbf{Z}_{f} & :=\left\{\mathbf{v} \in \mathbf{X}_{f}: b_{f}(\mathbf{v}, q)+b_{f, B}\left(\mathbf{v}, \beta_{f}\right)=0, \forall \beta_{f} \in \mathbb{R}^{K_{f}}, \forall q \in L^{2}\left(\Omega_{f}\right)\right\} .
\end{aligned}
$$

Lemma 1. There exists a constant $C>0$ such that

$$
\inf _{\beta_{f} \in \mathbb{R}^{K_{f}}, q \in L^{2}\left(\Omega_{f}\right)} \sup _{\mathbf{v} \in \mathbf{X}_{f}} \frac{b_{f}(\mathbf{v}, q)+b_{f, B}\left(\mathbf{v}, \beta_{f}\right)}{\|\mathbf{v}\|_{\mathbf{X}_{f}}\left(\|q\|_{L^{2}\left(\Omega_{f}\right)}+\left|\beta_{f}\right|\right)} \geq C .
$$

Proof. Taking advantage of the fact that $\mathbb{R}^{K_{f}}$ is finite-dimensional, we easily conclude

$$
\inf _{\beta_{f} \in \mathbb{R}^{K_{f}}} \sup _{\mathbf{v} \in \mathbf{X}_{f}} \frac{b_{f, B}\left(\mathbf{v}, \beta_{f}\right)}{\|\mathbf{v}\|_{\mathbf{X}_{f}}\left|\beta_{f}\right|} \geq C>0 .
$$

That is, for each $j=1, \cdots, K_{f}$, we find a $\mathbf{v}^{(j)} \in \mathbf{X}_{f}$ such as $\left.\mathbf{v}^{(j)}\right|_{\bigcup_{k \neq j} \Gamma_{f, k}}=\mathbf{0}$ and $\int_{\Gamma_{f, j}} v_{i}^{(j)} n_{i} d s \neq$ 0 . These $\mathbf{v}^{(j)}$ 's must exist because $\Gamma_{k, j}$ 's are pair-wise disjointed. It is then a trivial exercise to construct a function $\mathbf{v}$ as a linear combination of these $\mathbf{v}^{(j)}$ 's for a given $\beta_{f} \in \mathbb{R}^{K_{f}}$ to achieve $b_{f, B}\left(\mathbf{v}, \beta_{f}\right) \geq C\|\mathbf{v}\|_{\mathbf{X}_{f}}\left|\beta_{f}\right|$.

It is well known that an inf-sup condition holds for $b_{f}(\mathbf{v}, q)$ on $\mathbf{Z}_{f, 0} \times L^{2}\left(\Omega_{f}\right)$. Since $\mathbf{Z}_{f, 0} \subset \mathbf{Z}_{f, B}$, we know there exists an inf-sup condition for $b_{f}(\mathbf{v}, q)$ on $\mathbf{Z}_{f, B} \times L^{2}\left(\Omega_{f}\right)$. We now recall the fact that the continuity of the bilinear forms and individual inf-sup conditions imply the combined infsup condition [11] (see also Theorem 18) and conclude the existence of an inf-sup condition for $b_{f}(\mathbf{v}, q)+b_{f, B}\left(\mathbf{v}, \beta_{f}\right)$.

Theorem 1. There exists a unique solution $\left(\mathbf{u}, p, \beta_{f}\right) \in \mathbf{X}_{f} \times L^{2}\left(\Omega_{f}\right) \times \mathbb{R}^{K_{f}}$ satisfying (3.18)-(3.19). In addition, there exists a constant $C>0$ such that

$$
\begin{array}{r}
\|\mathbf{u}\|_{\mathbf{X}_{f}}+\sqrt{\sum_{m=1}^{d-1}\left\|\sqrt{\alpha_{S}} u_{i} t_{m i}\right\|_{L^{2}\left(\Gamma_{I}\right)}^{2}}+\|p\|_{L^{2}\left(\Omega_{f}\right)}+\left|\beta_{f}\right| \\
\leq C\left(\|\mathbf{f}\|_{\mathbf{X}_{f}^{*}}+\left\|\boldsymbol{\tau}_{B}\right\|_{\left(H^{-\frac{1}{2}\left(\Gamma_{f, N}\right)}\right)}{ }^{d}+\left\|p_{I}\right\|_{H^{-\frac{1}{2}\left(\Gamma_{I}\right)}}+\left|q_{f}\right|\right) .
\end{array}
$$

Proof. The existence and uniqueness of a solution follows from the continuity and strict monotonicity of $a_{f}(\cdot, \cdot)$ on $\mathbf{Z}_{f} \times \mathbf{Z}_{f}$, together with the inf-sup condition (3.20).

From the weak form (3.18), the inf-sup condition (3.20), and the assumption that $\nu_{f}(|D \mathbf{u}|)$ is 
bounded from above, we have

$$
\begin{aligned}
\|p\|_{L^{2}\left(\Omega_{f}\right)}+\left|\beta_{f}\right| & \leq C \sup _{\mathbf{v} \in \mathbf{X}_{f}} \frac{b_{f}(\mathbf{v}, p)+b_{f, B}\left(\mathbf{v}, \beta_{f}\right)}{\|\mathbf{v}\|_{\mathbf{X}_{f}}} \\
& =C \sup _{\mathbf{v} \in \mathbf{X}_{f}} \frac{a_{f}(\mathbf{u}, \mathbf{v})-l_{f}(\mathbf{v})}{\|\mathbf{v}\|_{\mathbf{X}_{f}}} \\
& \leq C\left(\|\mathbf{u}\|_{\mathbf{X}_{f}}+\|\mathbf{f}\|_{\mathbf{X}_{f}^{*}}+\left\|\boldsymbol{\tau}_{B}\right\|_{\left(H^{-\frac{1}{2}}\left(\Gamma_{f, N}\right)\right)^{d}}+\left\|p_{I}\right\|_{H^{-\frac{1}{2}\left(\Gamma_{I}\right)}}\right) .
\end{aligned}
$$

We choose $\mathbf{v}=\mathbf{u}, q=p$, and $\gamma_{f}=\beta_{f}$, and add (3.18)-(3.19) together to obtain

$$
\begin{aligned}
a_{f}(\mathbf{u}, \mathbf{u}) & =l_{f}(\mathbf{u})+b_{f, B}\left(q_{f} \mathbf{n}_{f}, \beta_{f}\right) \\
& =\left(f_{i}, u_{i}\right)_{\Omega_{f}}+\left\langle\boldsymbol{\tau}_{B}, u\right\rangle_{\Gamma_{f, N}}-\left\langle p_{I}, \mathbf{u} \cdot \mathbf{n}\right\rangle_{\Gamma_{I}}+\int_{\Gamma_{f, F}} \beta_{f} q_{f} d s \\
& \leq C\|\mathbf{u}\|_{\mathbf{X}_{f}}\left(\|\mathbf{f}\|_{\mathbf{X}_{f}^{*}}+\left\|\boldsymbol{\tau}_{B}\right\|_{\left(H^{-\frac{1}{2}}\left(\Gamma_{f, N}\right)\right)^{d}}+\left\|p_{I}\right\|_{H^{-\frac{1}{2}}\left(\Gamma_{I}\right)}\right) \\
& +C\left|\beta_{f}\right|\left|q_{f}\right| .
\end{aligned}
$$

Due to the assumption that meas $\left(\Gamma_{f, D}\right)>0$ and $\mathbf{u}_{B}=0$, we know from Korn's inequality:

$$
\|\mathbf{u}\|_{\mathbf{X}_{f}}=\|\mathbf{u}\|_{\left(H^{1}\left(\Omega_{f}\right)\right)^{d}} \leq C\|D \mathbf{u}\|_{\left(L^{2}\left(\Omega_{f}\right)\right)^{d \times d}},
$$

which implies the strict positivity of $a_{f}(\mathbf{u}, \mathbf{u})$ :

$$
\begin{aligned}
a_{f}(\mathbf{u}, \mathbf{u}) & =\int_{\Omega_{f}} 2 \nu(|D \mathbf{u}|)(D \mathbf{u})_{i j}(D \mathbf{u})_{i j} d x+\sum_{m=1}^{d-1} \int_{\Gamma_{S}} \alpha_{S}\left(u_{i} t_{m i}\right)\left(u_{k} t_{m k}\right) d s \\
& \geq 2 \nu_{\min }\|D \mathbf{u}\|_{\left(L^{2}\left(\Omega_{f}\right)\right)^{d \times d}}^{2}+\sum_{m=1}^{d-1}\left\|\sqrt{\alpha_{S}} u_{i} t_{m i}\right\|_{L^{2}\left(\Gamma_{I}\right)}^{2} \\
& \geq C\|\mathbf{u}\|_{\mathbf{x}_{f}}^{2}+\sum_{m=1}^{d-1}\left\|\sqrt{\alpha_{S}} u_{i} t_{m i}\right\|_{L^{2}\left(\Gamma_{I}\right)}^{2} .
\end{aligned}
$$

We then have

$$
\begin{aligned}
& \|\mathbf{u}\|_{\mathbf{X}_{f}}^{2}+\sum_{m=1}^{d-1}\left\|\sqrt{\alpha_{S}} u_{i} t_{m i}\right\|_{L^{2}\left(\Gamma_{I}\right)}^{2} \\
& \leq C\left(\|\mathbf{f}\|_{\mathbf{X}_{f}^{*}}^{2}+\left\|\boldsymbol{\tau}_{B}\right\|_{\left(H^{\left.-\frac{1}{2}\left(\Gamma_{f, N}\right)\right)}\right.}^{2}+\left\|p_{I}\right\|_{H^{-\frac{1}{2}\left(\Gamma_{I}\right)}}^{2}+\left|q_{f}\right|^{2}\right)+\epsilon\left|\beta_{f}\right|^{2} .
\end{aligned}
$$

The theorem follows from (3.23) and (3.24).

Remark: (See [11]) For sufficiently smooth data, f, $\boldsymbol{\tau}_{B}, p_{I}$ and solution $\mathbf{u}, p$, with

$$
\boldsymbol{\sigma} \mathbf{n}_{f}=s_{n} \mathbf{n}_{f}+\mathbf{s}_{T} \text {, where } s_{n}=\left(\boldsymbol{\sigma} \mathbf{n}_{f}\right) \cdot \mathbf{n}_{f} \text { and } \mathbf{s}_{T}=\boldsymbol{\sigma} \mathbf{n}_{f}-s_{n} \mathbf{n}_{f},
$$


and

$$
\left\langle\boldsymbol{\tau}_{B}, \mathbf{v}\right\rangle_{\Gamma_{f, N}}:=\int_{\Gamma_{f, N}} \boldsymbol{\tau}_{B, i} v_{i} d s,\left\langle p_{I}, \mathbf{v} \cdot \mathbf{n}_{f}\right\rangle_{\Gamma_{I}}:=\int_{\Gamma_{I}} p_{I} v_{i} n_{i} d s
$$

the unique solution of (3.18)-(3.19) satisfies

$$
-p+s_{n}=-\beta_{f, k} \quad \text { and } \quad \mathbf{s}_{T}=\mathbf{0} \quad \text { on } \Gamma_{f, k}, k=1,2, \ldots, K_{f} .
$$

Thus the variational form (3.18)-(3.19) corresponds to the boundary value problem (2.4)-(2.10) with the additional constraint (3.25).

\subsection{The reduced Stokes problem on the interface}

We let $\mathbf{f} \in \mathbf{X}_{f}^{*}, \boldsymbol{\tau}_{B} \in\left(H^{-\frac{1}{2}}\left(\Gamma_{f, N}\right)\right)^{d}$, and $q_{F} \in \mathbb{R}^{K_{F}}$ be fixed given data as before, but we consider $p_{I} \in H^{\frac{1}{2}}\left(\Gamma_{I}\right) \subset L^{2}\left(\Gamma_{I}\right)$ as a variable input. From Theorem 1, we know that there exists a unique solution $\left(\mathbf{u}, p, \beta_{f}\right) \in \mathbf{X}_{f} \times L^{2}\left(\Omega_{f}\right) \times \mathbb{R}^{K_{F}}$ of (3.18)-(3.19) that is a function of $p_{I}$. We denote the solution as $\left(\mathbf{u}^{*}\left(p_{I}\right), p^{*}\left(p_{I}\right), \beta_{f}^{*}\left(p_{I}\right)\right)$. Before studying the relationship between $\mathbf{u}^{*}\left(p_{I}\right)$ and $p_{I}$, we need a lemma, which we use below in the proof of Theorem 2 :

Lemma 2. If meas $\left(\Gamma_{f, N}\right)>0$, then there exists a constant $C>0$ such that for all $\lambda \in H^{-\frac{1}{2}}\left(\Gamma_{I}\right)$ :

$$
\sup _{\mathbf{v} \in \mathbf{X}_{f}} \frac{\langle\lambda, \mathbf{v} \cdot \mathbf{n}\rangle_{\Gamma_{I}}}{\|\mathbf{v}\|_{\mathbf{X}_{f}}} \leq C \sup _{\mathbf{v} \in \mathbf{Z}_{f}} \frac{\langle\lambda, \mathbf{v} \cdot \mathbf{n}\rangle_{\Gamma_{I}}}{\|\mathbf{v}\|_{\mathbf{X}_{f}}}
$$

As a result, we have

$$
\frac{1}{C}\|\lambda\|_{H^{-\frac{1}{2}}\left(\Gamma_{I}\right)} \leq \sup _{\mathbf{v} \in \mathbf{Z}_{f}} \frac{\langle\lambda, \mathbf{v} \cdot \mathbf{n}\rangle_{\Gamma_{I}}}{\|\mathbf{v}\|_{\mathbf{X}_{f}}} \leq\|\lambda\|_{H^{-\frac{1}{2}\left(\Gamma_{I}\right)}}
$$

Proof. We first note two inf-sup conditions:

$$
\inf _{\lambda \in H^{-\frac{1}{2}}\left(\Gamma_{I}\right)} \sup _{\mathbf{v} \in \mathbf{X}_{f}} \frac{\langle\lambda, \mathbf{v} \cdot \mathbf{n}\rangle_{\Gamma_{I}}}{\|\mathbf{v}\|_{\mathbf{X}_{f}}\|\lambda\|_{H^{-\frac{1}{2}}\left(\Gamma_{I}\right)}} \geq C>0,
$$

and

$$
\inf _{\beta_{f} \in \mathbb{R}^{K_{f}}} \sup _{\mathbf{v} \in \mathbf{Z}_{f}^{\Gamma_{I}}} \frac{b_{f, B}\left(\mathbf{v}, \beta_{f}\right)}{\|\mathbf{v}\|_{\mathbf{X}_{f}}\left|\beta_{f}\right|} \geq C>0
$$

where

$$
\mathbf{Z}_{f}^{\Gamma_{I}}:=\left\{\mathbf{v} \in \mathbf{X}_{f}:\langle\lambda, \mathbf{v} \cdot \mathbf{n}\rangle_{\Gamma_{I}}=0, \forall \lambda \in H^{-\frac{1}{2}}\left(\Gamma_{I}\right)\right\}
$$

Inequality (3.26) is well known, and (3.27) can be established by taking advantage of the finite dimensionality of $\mathbb{R}^{K_{f}}$ as we did for Lemma 1. Theorem 18 and (3.26)-(3.27) imply

$$
\inf _{\lambda \in H^{-\frac{1}{2}}\left(\Gamma_{I}\right), \beta_{f} \in \mathbb{R}^{K_{f}}} \sup _{\mathbf{v} \in \mathbf{X}_{f}} \frac{\langle\lambda, \mathbf{v} \cdot \mathbf{n}\rangle_{\Gamma_{I}}+b_{f, B}\left(\mathbf{v}, \beta_{f}\right)}{\|\mathbf{v}\|_{\mathbf{X}_{f}}\left(\|\lambda\|_{H^{-\frac{1}{2}}\left(\Gamma_{I}\right)}+\left|\beta_{f}\right|\right)} \geq C>0 .
$$


As meas $\left(\Gamma_{f, N}\right)>0$, we can show the following inf-sup condition by applying an argument similar to the one used to prove Lemma 1:

$$
\inf _{q \in L^{2}\left(\Omega_{f}\right)} \sup _{\mathbf{v} \in \mathbf{Z}_{f}^{b_{f, B}, \Gamma_{I}}} \frac{b_{f}(\mathbf{v}, q)}{\|\mathbf{v}\|_{\mathbf{X}_{f}}\|q\|_{L^{2}\left(\Omega_{f}\right)}} \geq C>0
$$

where

$$
\mathbf{Z}_{f}^{b_{f, B}, \Gamma_{I}}:=\left\{\mathbf{v} \in \mathbf{X}_{f}:\langle\lambda, \mathbf{v} \cdot \mathbf{n}\rangle_{\Gamma_{I}}+b_{f, B}\left(\mathbf{v}, \beta_{f}\right)=0, \forall \lambda \in H^{-\frac{1}{2}}\left(\Gamma_{I}\right), \forall \beta_{f} \in \mathbb{R}^{K_{f}}\right\} .
$$

Now Theorem 18 and (3.28)-(3.29) imply

$$
\inf _{\lambda \in H^{-\frac{1}{2}}\left(\Gamma_{I}\right), q \in L^{2}\left(\Omega_{f}\right), \beta_{f} \in \mathbb{R}^{K_{f}}} \sup _{\mathbf{v} \in \mathbf{X}_{f}} \frac{\langle\lambda, \mathbf{v} \cdot \mathbf{n}\rangle_{\Gamma_{I}}+b_{f}(\mathbf{v}, q)+b_{f, B}\left(\mathbf{v}, \beta_{f}\right)}{\|\mathbf{v}\|_{\mathbf{X}_{f}}\left(\|\lambda\|_{H^{-\frac{1}{2}\left(\Gamma_{I}\right)}}+\|q\|_{L^{2}\left(\Omega_{f}\right)}+\left|\beta_{f}\right|\right)} \geq C>0 .
$$

Applying Theorem 18 again, we see that (3.30) leads to

$$
\inf _{\lambda \in H^{-\frac{1}{2}}\left(\Gamma_{I}\right)} \sup _{\mathbf{v} \in \mathbf{Z}_{f}} \frac{\langle\lambda, \mathbf{v} \cdot \mathbf{n}\rangle_{\Gamma_{I}}}{\|\mathbf{v}\|_{\mathbf{X}_{f}}\|\lambda\|_{H^{-\frac{1}{2}}\left(\Gamma_{I}\right)}} \geq C>0
$$

or equivalently,

$$
\|\lambda\|_{H^{-\frac{1}{2}\left(\Gamma_{I}\right)}} \leq \frac{1}{C} \sup _{\mathbf{v} \in \mathbf{Z}_{f}} \frac{\langle\lambda, \mathbf{v} \cdot \mathbf{n}\rangle_{\Gamma_{I}}}{\|\mathbf{v}\|_{\mathbf{X}_{f}}}, \quad \forall \lambda \in H^{-\frac{1}{2}}\left(\Gamma_{I}\right) .
$$

Theorem 2. There exists a constant $C>0$ such that for all $\lambda \in H^{-\frac{1}{2}}\left(\Gamma_{I}\right)$ and $\mu \in H^{-\frac{1}{2}}\left(\Gamma_{I}\right)$,

$$
\left\|\mathbf{u}^{*}(\lambda)-\mathbf{u}^{*}(\mu)\right\|_{\mathbf{X}_{f}} \leq C\|\lambda-\mu\|_{H^{-\frac{1}{2}}\left(\Gamma_{I}\right)} .
$$

If meas $\left(\Gamma_{f, N}\right)>0$, we further have

$$
\frac{1}{C}\|\lambda-\mu\|_{H^{-\frac{1}{2}\left(\Gamma_{I}\right)}} \leq\left\|\mathbf{u}^{*}(\lambda)-\mathbf{u}^{*}(\mu)\right\|_{\mathbf{X}_{f}} \leq C\|\lambda-\mu\|_{H^{-\frac{1}{2}\left(\Gamma_{I}\right)}} .
$$

Proof. To show (3.31), we pick $\mathbf{u}=\mathbf{u}^{*}(\lambda), \mathbf{v}=\mathbf{u}^{*}(\mu), p=p^{*}(\lambda)$, and $\beta_{f}=\beta_{f}^{*}(\lambda)$ in (3.18), and pick $\mathbf{u}=\mathbf{u}^{*}(\mu), q=p^{*}(\lambda)$, and $\gamma_{f}=\beta_{f}^{*}(\lambda)$ in (3.19), and then add them together to obtain

$$
a_{f}\left(\mathbf{u}^{*}(\lambda), \mathbf{u}^{*}(\mu)\right)=l_{f}\left(\mathbf{u}^{*}(\mu)\right)+b_{f, B}\left(q_{f} \mathbf{n}_{f}, \beta_{f}^{*}(\lambda)\right) .
$$

The strict monotonicity of $a_{f}(\cdot, \cdot)$ then implies

$$
\begin{aligned}
\frac{1}{C}\left\|\mathbf{u}^{*}(\lambda)-\mathbf{u}^{*}(\mu)\right\|_{\mathbf{X}_{f}}^{2} & \leq a_{f}\left(\mathbf{u}^{*}(\lambda), \mathbf{u}^{*}(\lambda)-\mathbf{u}^{*}(\mu)\right)-a_{f}\left(\mathbf{u}^{*}(\mu), \mathbf{u}^{*}(\lambda)-\mathbf{u}^{*}(\mu)\right) \\
& =-\left\langle(\lambda-\mu),\left(\mathbf{u}^{*}(\lambda)-\mathbf{u}^{*}(\mu)\right) \cdot \mathbf{n}_{f}\right\rangle_{\Gamma_{I}} \\
& \leq C\left\|\mathbf{u}^{*}(\lambda)-\mathbf{u}^{*}(\mu)\right\|_{\mathbf{X}_{f}}\|\lambda-\mu\|_{H^{-\frac{1}{2}}\left(\Gamma_{I}\right)}
\end{aligned}
$$

which yields the desired bound in (3.31). 
To show the first inequality in (3.32), we consider

$$
\begin{aligned}
-a_{f}\left(\mathbf{u}^{*}(\lambda), \mathbf{v}\right)+a_{f}\left(\mathbf{u}^{*}(\mu), \mathbf{v}\right)+b_{f}\left(\mathbf{v}, p^{*}(\lambda)-p^{*}(\mu)\right)+b_{f, B}\left(\mathbf{v}, \beta_{f}^{*}(\lambda)-\beta_{f}^{*}(\mu)\right) & \\
& =\left\langle(\lambda-\mu), \mathbf{v} \cdot \mathbf{n}_{f}\right\rangle_{\Gamma_{I}} .
\end{aligned}
$$

For $\mathbf{v} \in \mathbf{Z}_{f} \subset \mathbf{X}_{f}$, we have

$$
\begin{aligned}
\left\langle(\lambda-\mu), \mathbf{v} \cdot \mathbf{n}_{f}\right\rangle_{\Gamma_{I}} & =-a_{f}\left(\mathbf{u}^{*}(\lambda), \mathbf{v}\right)+a_{f}\left(\mathbf{u}^{*}(\mu), \mathbf{v}\right) \\
& \leq C\left\|\mathbf{u}^{*}(\lambda)-\mathbf{u}^{*}(\mu)\right\|_{\mathbf{X}_{f}}\|\mathbf{v}\|_{\mathbf{X}_{f}} .
\end{aligned}
$$

Consequently, using Lemma 2 and (3.33)

$$
\begin{aligned}
\|\lambda-\mu\|_{H^{-\frac{1}{2}}\left(\Gamma_{I}\right)} & =\sup _{q \in H^{\frac{1}{2}}\left(\Gamma_{I}\right)} \frac{\langle(\lambda-\mu), q\rangle)_{\Gamma_{I}}}{\|q\|_{H^{\frac{1}{2}}\left(\Gamma_{I}\right)}}=\sup _{\mathbf{v} \in \mathbf{X}_{f}} \frac{\left\langle(\lambda-\mu), \mathbf{v} \cdot \mathbf{n}_{f}\right\rangle_{\Gamma_{I}}}{\|\mathbf{v} \cdot \mathbf{n}\|_{H^{\frac{1}{2}}\left(\Gamma_{I}\right)}} \\
& \leq C \sup _{\mathbf{v} \in \mathbf{Z}_{f}} \frac{\left\langle(\lambda-\mu), \mathbf{v} \cdot \mathbf{n}_{f}\right\rangle_{\Gamma_{I}}}{\|\mathbf{v} \cdot \mathbf{n}\|_{H^{\frac{1}{2}}\left(\Gamma_{I}\right)}} \leq C\left\|\mathbf{u}^{*}(\lambda)-\mathbf{u}^{*}(\mu)\right\|_{\mathbf{X}_{f}} .
\end{aligned}
$$

If the normal velocity component $q_{I} \in H^{\frac{1}{2}}\left(\Gamma_{I}\right)$ is given on the interface $\Gamma_{I}$, rather than the pressure, we can solve for the pressure by seeking $\lambda \in H^{-\frac{1}{2}}\left(\Gamma_{I}\right)$ such that

$$
A_{f}(\lambda, \mu)=-\left\langle\mu, q_{I}\right\rangle_{\Gamma_{I}} \quad \forall \mu \in H^{-\frac{1}{2}}\left(\Gamma_{I}\right),
$$

with the form $A_{f}(\cdot, \cdot)$ defined by

$$
A_{f}(\lambda, \mu):=-\left\langle\mu, \mathbf{u}_{f}^{*}(\lambda) \cdot \mathbf{n}_{f}\right\rangle_{\Gamma_{I}}
$$

We now prove a few properties of $A_{f}(\cdot, \cdot)$.

Theorem 3. $A_{f}(\lambda, \mu)$ is a nonlinear functional of $\lambda$ and a linear functional of $\mu . A_{f}(\cdot, \cdot)$ is continuous on $H^{-\frac{1}{2}}\left(\Gamma_{I}\right) \times H^{-\frac{1}{2}}\left(\Gamma_{I}\right)$. In addition, $A_{f}(\cdot, \cdot)$ is monotone on $H^{-\frac{1}{2}}\left(\Gamma_{I}\right) \times H^{-\frac{1}{2}}\left(\Gamma_{I}\right)$. If $\operatorname{meas}\left(\Gamma_{f, N}\right)>0, A_{f}(\cdot, \cdot)$ is strictly monotone on $H^{-\frac{1}{2}}\left(\Gamma_{I}\right) \times H^{-\frac{1}{2}}\left(\Gamma_{I}\right)$.

Proof. It is clear that $A_{f}(\lambda, \mu)$ is linear in $\mu$. Its continuity follows from Theorem 2 . We now show the strict monotonicity assuming meas $\left(\Gamma_{f, N}\right)>0$. From the weak formulation (3.18), we know

$$
\begin{aligned}
A_{f}(\lambda, \mu)= & -\left\langle\mu, \mathbf{u}_{f}^{*}(\lambda) \cdot \mathbf{n}_{f}\right\rangle_{\Gamma_{I}} \\
= & a_{f}\left(\mathbf{u}^{*}(\mu), \mathbf{u}^{*}(\lambda)\right)-b_{f}\left(\mathbf{u}^{*}(\lambda), p^{*}(\mu)\right)-b_{f, B}\left(\mathbf{u}^{*}(\lambda), \beta_{f}^{*}(\mu)\right) \\
& \quad-\left(\mathbf{f}, \mathbf{u}^{*}(\lambda)\right)_{\Omega_{f}}-\left\langle\boldsymbol{\tau}_{B}, \mathbf{u}_{f}^{*}(\lambda)\right\rangle_{\Gamma_{f, N}} \\
= & a_{f}\left(\mathbf{u}^{*}(\mu), \mathbf{u}^{*}(\lambda)\right)-b_{f, B}\left(q_{f} \mathbf{n}_{f}, \beta_{f}^{*}(\mu)\right)-\left(\mathbf{f}, \mathbf{u}^{*}(\lambda)\right)_{\Omega_{f}}-\left\langle\boldsymbol{\tau}_{B}, \mathbf{u}_{f}^{*}(\lambda)\right\rangle_{\Gamma_{f, N}} .
\end{aligned}
$$

We then obtain

$$
\begin{aligned}
A_{f}(\lambda, \lambda-\mu)-A_{f}(\mu, \lambda-\mu) & =A_{f}(\lambda, \lambda)-A_{f}(\lambda, \mu)-A_{f}(\mu, \lambda)+A_{f}(\mu, \mu) \\
& =a_{f}\left(\mathbf{u}^{*}(\lambda), \mathbf{u}^{*}(\lambda)-\mathbf{u}^{*}(\mu)\right)-a_{f}\left(\mathbf{u}^{*}(\mu), \mathbf{u}^{*}(\lambda)-\mathbf{u}^{*}(\mu)\right) \\
& \geq C\left\|\mathbf{u}^{*}(\lambda)-\mathbf{u}^{*}(\mu)\right\|_{\left(H^{1}\left(\Omega_{f}\right)\right)^{d}}^{2} \\
& \geq C\|\lambda-\mu\|_{H^{-\frac{1}{2}}\left(\Gamma_{I}\right)}^{2},
\end{aligned}
$$


where we used Theorem 2 to obtain the last inequality above. If meas $\left(\Gamma_{f, N}\right)=0$, the last inequality above may fail to hold, and we then have only monotonicity (not strict monotonicity).

Theorem 4. If meas $\left(\Gamma_{f, N}\right)>0$, then there is a unique solution $\lambda \in H^{-\frac{1}{2}}\left(\Gamma_{I}\right)$ to the reduced problem (3.34) for any given $q_{I} \in H^{\frac{1}{2}}\left(\Gamma_{I}\right)$.

Proof. This theorem follows directly from the continuity and the strict monotonicity of $A_{f}(\cdot, \cdot)$.

\subsection{Darcy flow}

We next restrict our attention to the Darcy flow problem, assuming that the pressure is specified on the interface. Specifically, we consider (2.11)-(2.15) together with:

$$
p=p_{I}, \quad \text { on } \Gamma_{I} .
$$

Multiplying (2.11) by a smooth vector function $\mathbf{v}$, with $\left.\mathbf{v} \cdot \mathbf{n}_{p}\right|_{\Gamma_{p, N}}=0$, integrate over $\Omega_{p}$ and apply the divergence theorem we obtain

$$
\begin{aligned}
\left(f_{i}, v_{i}\right)_{\Omega_{p}}= & \int_{\Omega_{p}}\left(\nu_{p}(|\mathbf{u}|) R_{i j} u_{j}+\frac{\partial p}{\partial x_{i}}\right) v_{i} d x \\
= & \int_{\Omega_{p}} \nu_{p}(|\mathbf{u}|) R_{i j} u_{j} v_{i} d x-\int_{\Omega_{p}} p \frac{\partial v_{i}}{\partial x_{i}} d x+\int_{\partial \Omega_{p}} p v_{i} n_{i} d x \\
= & \int_{\Omega_{p}} \nu_{p}(|\mathbf{u}|) R_{i j} u_{j} v_{i} d x-\int_{\Omega_{p}} p \frac{\partial v_{i}}{\partial x_{i}} d x+\int_{\Gamma_{p, D}} p_{B} v_{i} n_{i} d s \\
& \quad+\int_{\Gamma_{p, F}} p v_{i} n_{i} d s+\int_{\Gamma_{I}} p_{I} v_{i} n_{i} d s .
\end{aligned}
$$

In part, to incorporate the specified flow rate constraints into the formulation, for each $\Gamma_{p, k}$ we replace $\int_{\Gamma_{p, k}} p v_{i} n_{i} d s$ by

$$
\begin{aligned}
\int_{\Gamma_{p, k}} p v_{i} n_{i} d s & \longrightarrow \int_{\Gamma_{p, k}} \beta_{p, k} v_{i} n_{i} d s, \beta_{p, k} \in \mathbb{R}, k=1, \ldots, K_{p}, \\
\text { equivalently } & \int_{\Gamma_{p, F}} p v_{i} n_{i} d s \longrightarrow \int_{\Gamma_{p, F}} \beta_{p} v_{i} n_{i} d s,
\end{aligned}
$$

where $\beta_{p} \in \mathbb{R}^{K_{p}}$ in (3.36) is interpreted as a piecewise constant function on $\Gamma_{p, F}$.

The averaged flow rates are imposed by

$$
\int_{\Gamma_{p, F}} \gamma_{p} u_{i} n_{i} d s=\sum_{k=1}^{K_{p}} \int_{\Gamma_{p, k}} \gamma_{p, k} q_{p, k} d s=\sum_{k=1}^{K_{p}} \gamma_{p, k} q_{p, k} \operatorname{meas}\left(\Gamma_{p, k}\right) .
$$

Similar to the previous treatment, the divergence-free equation (2.12) is weakly imposed using

$$
\int_{\Omega_{p}} \frac{\partial u_{i}}{\partial x_{i}} q=0, \quad \forall q \in L^{2}\left(\Omega_{p}\right) .
$$


For $\mathbf{u} \in H\left(\operatorname{div}, \Omega_{p}\right), \mathbf{u} \cdot \mathbf{n}_{p} \in H^{-1 / 2}\left(\partial \Omega_{p}\right)$. For $p \in H^{1 / 2}\left(\Gamma_{I}\right)$ duality pairing does not define $\left\langle\mathbf{u} \cdot \mathbf{n}_{p}, p_{I}\right\rangle_{\Gamma_{I}}$, as $\mathbf{u} \cdot \mathbf{n}_{p}$ acts on functions in $H^{1 / 2}\left(\partial \Omega_{p}\right)$. Following the work of Galvis and Sarkis ([14], Lemma 2.1), given $\Gamma_{s} \subset \partial \Omega_{p}, r \in H^{1 / 2}\left(\Gamma_{s}\right)$, let $E_{\Gamma_{s}}^{1 / 2} r \in H^{1 / 2}\left(\partial \Omega_{p}\right)$ denote the extension of $r$ to $\partial \Omega_{p}$. Then, for $f \in H^{-1 / 2}\left(\partial \Omega_{p}\right)$, we denote

$$
\langle f, r\rangle_{\Gamma_{s}}:=\left\langle f, E_{\Gamma_{s}}^{1 / 2} r\right\rangle_{\partial \Omega_{p}} .
$$

Also, as given in [14], for $f \in H^{-1 / 2}\left(\partial \Omega_{p}\right),\left.f\right|_{\Gamma_{s}}=0$ is defined as

$$
\left\langle f, E_{00, \Gamma_{s}}^{1 / 2} w\right\rangle_{\partial \Omega_{p}}=0, \text { for all } w \in H_{00}^{1 / 2}\left(\Gamma_{s}\right),
$$

where $E_{00, \Gamma_{s}}^{1 / 2} w$ denotes the extension by 0 of $w$ to $\partial \Omega_{p} \backslash \Gamma_{s}$.

We now introduce

$$
\begin{aligned}
\mathbf{X}_{p}=\left\{\mathbf{v}: \mathbf{v} \in \mathbf{H}\left(\operatorname{div}, \Omega_{p}\right),\left.\mathbf{v} \cdot \mathbf{n}\right|_{\Gamma_{p, N}}=0\right\} \\
a_{p}(\mathbf{u}, \mathbf{v}):=\int_{\Omega_{p}} \nu(|\mathbf{u}|) R_{i j} u_{j} v_{i} d x \\
b_{p}(\mathbf{v}, p):=\int_{\Omega_{p}} \frac{\partial v_{i}}{\partial x_{i}} p d x \\
b_{p, B}\left(\mathbf{v}, \beta_{p}\right):=-\left\langle\mathbf{v} \cdot \mathbf{n}_{p}, \beta_{p}\right\rangle_{\Gamma_{p, F}}, \\
l_{p}(\mathbf{v}):=\left(f_{i}, v_{i}\right)_{\Omega_{p}}-\left\langle\mathbf{v} \cdot \mathbf{n}_{p}, p_{B}\right\rangle_{\Gamma_{p, D}}-\left\langle\mathbf{v} \cdot \mathbf{n}_{p}, p_{I}\right\rangle_{\Gamma_{I}}
\end{aligned}
$$

The weak formulation for the Darcy flow is: Given $\mathbf{f} \in \mathbf{X}_{p}^{*}, p_{B} \in H^{\frac{1}{2}}\left(\Gamma_{p, D}\right), q_{p} \in \mathbb{R}^{K_{p}}$, and $p_{I} \in H^{\frac{1}{2}}\left(\Gamma_{I}\right)$, determine $\left(\mathbf{u}, p, \beta_{p}\right) \in \mathbf{X}_{p} \times L^{2}\left(\Omega_{p}\right) \times \mathbb{R}^{K_{p}}$ such that

$$
\begin{aligned}
a_{p}(\mathbf{u}, \mathbf{v})-b_{p}(\mathbf{v}, p)-b_{p, B}\left(\mathbf{v}, \beta_{p}\right) & =l_{p}(\mathbf{v}), \quad \forall \mathbf{v} \in \mathbf{X}_{p}, \\
b_{p}(\mathbf{u}, q)+b_{p, B}\left(\mathbf{u}, \gamma_{p}\right) & =b_{p, B}\left(q_{p} \mathbf{n}_{p}, \gamma_{p}\right), \quad \forall\left(q, \gamma_{p}\right) \in L^{2}\left(\Omega_{p}\right) \times \mathbb{R}^{K_{p}} .
\end{aligned}
$$

Before presenting our results, we introduce

$$
\begin{aligned}
\mathbf{Z}_{p, B} & :=\left\{\mathbf{v} \in \mathbf{X}_{p}: b_{f, B}\left(\mathbf{v}, \beta_{p}\right)=0, \forall \beta_{p} \in \mathbb{R}^{K_{p}}\right\}, \\
\mathbf{Z}_{p, 0} & :=\left\{\mathbf{v} \in \mathbf{X}_{p}:\left.\mathbf{v} \cdot \mathbf{n}\right|_{\Gamma_{p, F}}=0\right\}, \\
\mathbf{Z}_{p} & :=\left\{\mathbf{v} \in \mathbf{X}_{p}: b_{p}(\mathbf{v}, q)+b_{p, B}\left(\mathbf{v}, \beta_{p}\right)=0, \forall \beta_{p} \in \mathbb{R}^{K_{p}}, \forall q \in L^{2}\left(\Omega_{f}\right)\right\} .
\end{aligned}
$$

Lemma 3. There exists a constant $C>0$ such that

$$
\inf _{\beta_{p} \in \mathbb{R}^{K_{p}, q \in L^{2}\left(\Omega_{f}\right)}} \sup _{\mathbf{v} \in \mathbf{X}_{p}} \frac{b_{p}(\mathbf{v}, q)+b_{p, B}\left(\mathbf{v}, \beta_{p}\right)}{\|\mathbf{v}\|_{\mathbf{X}_{p}}\left(\|q\|_{L^{2}\left(\Omega_{f}\right)}+\left|\beta_{p}\right|\right)} \geq C .
$$

Proof. Similar to our previous arguments, we can utilize the finite dimensionality of $\mathbb{R}^{K_{p}}$ to obtain the following inf-sup condition:

$$
\inf _{\beta_{p} \in \mathbb{R}^{K_{p}}} \sup _{\mathbf{v} \in \mathbf{X}_{p}} \frac{b_{p, B}\left(\mathbf{v}, \beta_{p}\right)}{\|\mathbf{v}\|_{\mathbf{X}_{p}}\left|\beta_{p}\right|} \geq C>0 .
$$


It is well known that an inf-sup condition holds for $b_{p}(\mathbf{v}, q)$ on $\mathbf{Z}_{p, 0} \times L^{2}\left(\Omega_{p}\right)$. Since $\mathbf{Z}_{p, 0} \subset \mathbf{Z}_{p, B}$, we know there exists an inf-sup condition for $b_{p}(\mathbf{v}, q)$ on $\mathbf{Z}_{p, B} \times L^{2}\left(\Omega_{p}\right)$. Theorem 18 then implies the existence of an inf-sup condition for $b_{p}(\mathbf{v}, q)+b_{p, B}\left(\mathbf{v}, \beta_{p}\right)$.

Theorem 5. There exists a unique solution $\left(\mathbf{u}, p, \beta_{p}\right) \in \mathbf{X}_{p} \times L^{2}\left(\Omega_{p}\right) \times \mathbb{R}^{K_{p}}$ satisfying (3.37)-(3.38). In addition, there exists a constant $C>0$ such that

$$
\|\mathbf{u}\|_{\mathbf{X}_{p}}+\|p\|_{L^{2}\left(\Omega_{p}\right)}+\left|\beta_{p}\right| \leq C\left(\|\mathbf{f}\|_{\mathbf{X}_{p}^{*}}+\left\|p_{B}\right\|_{H^{\frac{1}{2}}\left(\Gamma_{p, D}\right)}+\left\|p_{I}\right\|_{H^{\frac{1}{2}\left(\Gamma_{I}\right)}}+\left|q_{p}\right|\right) .
$$

Proof. The existence and uniqueness of the solution follows from the continuity and strict monotonicity of $a_{p}(\cdot, \cdot)$ on $\mathbf{Z}_{p} \times \mathbf{Z}_{p}$ and the inf-sup condition (3.39).

From the weak formulation (3.37), the inf-sup condition (3.39) and the assumption that $\nu_{p}(|\mathbf{u}|)$ and $\mathbf{R}$ are both bounded from above, we have

$$
\begin{aligned}
\|p\|_{L^{2}\left(\Omega_{p}\right)}+\left|\beta_{p}\right| & \leq C \sup _{\mathbf{v} \in \mathbf{X}_{p}} \frac{b_{p}(\mathbf{v}, p)+b_{p, B}\left(\mathbf{v}, \beta_{p}\right)}{\|\mathbf{v}\|_{\mathbf{X}_{p}}} \\
& =C \sup _{\mathbf{v} \in \mathbf{X}_{p}} \frac{a_{p}(\mathbf{u}, \mathbf{v})-l_{p}(\mathbf{v})}{\|\mathbf{v}\|_{\mathbf{X}_{p}}} \\
& \leq C\left(\|\mathbf{u}\|_{\mathbf{X}_{p}}+\|\mathbf{f}\|_{\mathbf{X}_{p}^{*}}+\left\|p_{B}\right\|_{H^{\frac{1}{2}\left(\Gamma_{p, D}\right)}}+\left\|p_{I}\right\|_{H^{\frac{1}{2}\left(\Gamma_{I}\right)}}\right) .
\end{aligned}
$$

We pick $q=\nabla \cdot \mathbf{u}$ and $\gamma_{p}=0$ in (3.38) to obtain

$$
\|\nabla \cdot \mathbf{u}\|_{L^{2}\left(\Omega_{p}\right)}^{2}=b_{p}(\mathbf{u}, \nabla \cdot \mathbf{u})=0,
$$

which implies that

$$
\|\mathbf{u}\|_{\mathbf{X}_{p}}=\|\mathbf{u}\|_{\mathbf{H}\left(\operatorname{div}, \Omega_{p}\right)}=\|\mathbf{u}\|_{\left(L^{2}\left(\Omega_{p}\right)\right)^{d}}
$$

To see the regularity inequality, we pick $\mathbf{v}=\mathbf{u}, q=p$, and $\gamma_{p}=\beta_{p}$, and add (3.37)-(3.38) together to obtain

$$
\begin{aligned}
a_{p}(\mathbf{u}, \mathbf{u}) & =l_{p}(\mathbf{u})+b_{p, B}\left(q_{p} \mathbf{n}_{p}, \beta_{p}\right) \\
& =\left(f_{i}, u_{i}\right)_{\Omega_{p}}-\left\langle\mathbf{u} \cdot \mathbf{n}_{p}, p_{B}\right\rangle_{\Gamma_{p, D}}-\left\langle\mathbf{u} \cdot \mathbf{n}_{p}, p_{I}\right\rangle_{\Gamma_{I}}-\left\langle q_{p} \mathbf{n}_{p}, \beta_{p}\right\rangle_{\Gamma_{p, F}} \\
& \leq C\left(\|\mathbf{f}\|_{\mathbf{X}_{p}^{*}}^{2}+\left\|p_{B}\right\|_{H^{\frac{1}{2}}\left(\Gamma_{p, D}\right)}^{2}+\left\|p_{I}\right\|_{H^{\frac{1}{2}\left(\Gamma_{I}\right)}}^{2}+\left|q_{p}\right|^{2}\right)+\epsilon\left(\|\mathbf{u}\|_{\mathbf{X}_{p}}^{2}+\left|\beta_{p}\right|^{2}\right) .
\end{aligned}
$$

From the assumptions that the viscosity $\nu_{p}(|\mathbf{u}|)$ is bounded from below and that $\mathbf{R}$ is strictly positive definite, we have

$$
a_{p}(\mathbf{u}, \mathbf{u})=\int_{\Omega_{p}} \nu_{p}(|\mathbf{u}|) R_{i j} u_{j} v_{i} d x \geq C\|\mathbf{u}\|_{\left(L^{2}\left(\Omega_{p}\right)\right)^{d}}
$$

Now the theorem follows from (3.41)-(3.43).

Remark: (See [11]) For sufficiently smooth data, $\mathbf{f}, p_{B}, p_{I}$ and solution $\mathbf{u}, p$ the unique solution of (3.37)-(3.38) satisfies

$$
p=-\beta_{p, k} \text { on } \Gamma_{p, k}, k=1,2, \ldots, K_{p} .
$$

Thus the variational form (3.37)-(3.38) corresponds to the boundary value problem (2.11)-(2.15) with the additional constraint (3.44). 


\subsection{The reduced Darcy problem on the interface}

Let $\mathbf{f} \in \mathbf{X}_{p}^{*}, p_{B} \in H^{\frac{1}{2}}\left(\Gamma_{p, D}\right)$, and $q_{p} \in \mathbb{R}^{K_{p}}$ be fixed given data as before. For each $p_{I} \in H^{\frac{1}{2}}\left(\Gamma_{I}\right) \subset$ $H^{-\frac{1}{2}}\left(\Gamma_{I}\right)$, we have a unique solution $\left(\mathbf{u}^{*}\left(p_{I}\right), p^{*}\left(p_{I}\right), \beta_{p}^{*}\left(p_{I}\right)\right) \in \mathbf{X}_{p} \times L^{2}\left(\Omega_{p}\right) \times \mathbb{R}^{K_{p}}$ that satisfies (3.37)-(3.38).

Theorem 6. There exists a constant $C>0$ such that

$$
\left\|\mathbf{u}^{*}(\lambda)-\mathbf{u}^{*}(\mu)\right\|_{\mathbf{X}_{p}} \leq C\|\lambda-\mu\|_{H^{\frac{1}{2}\left(\Gamma_{I}\right)}} .
$$

If meas $\left(\Gamma_{p, D}\right)>0$, we further have

$$
\frac{1}{C}\|\lambda-\mu\|_{H^{\frac{1}{2}\left(\Gamma_{I}\right)}} \leq\left\|\mathbf{u}^{*}(\lambda)-\mathbf{u}^{*}(\mu)\right\|_{\mathbf{X}_{p}} \leq C\|\lambda-\mu\|_{H^{\frac{1}{2}\left(\Gamma_{I}\right)}} .
$$

Proof. To show the first inequality in (3.46), we consider

$$
\begin{array}{r}
-a_{p}\left(\mathbf{u}^{*}(\lambda), \mathbf{v}\right)+a_{p}\left(\mathbf{u}^{*}(\mu), \mathbf{v}\right)+b_{p}\left(\mathbf{v}, p^{*}(\lambda)-p^{*}(\mu)\right)+b_{p, B}\left(\mathbf{v}, \beta_{p}^{*}(\lambda)-\beta_{p}^{*}(\mu)\right) \\
=\left\langle\mathbf{v} \cdot \mathbf{n}_{p},(\lambda-\mu)\right\rangle_{\Gamma_{I}} .
\end{array}
$$

For $\mathbf{v} \in \mathbf{Z}_{p} \subset \mathbf{X}_{p}$

$$
\begin{aligned}
C\left\|\mathbf{u}^{*}(\lambda)-\mathbf{u}^{*}(\mu)\right\|_{\mathbf{X}_{p}}\|\mathbf{v}\|_{\mathbf{X}_{p}} & =C\left\|\mathbf{u}^{*}(\lambda)-\mathbf{u}^{*}(\mu)\right\|_{\left(L^{2}\left(\Omega_{p}\right)\right)^{d}}\|\mathbf{v}\|_{\left(L^{2}\left(\Omega_{p}\right)\right)^{d}} \\
& \geq-a_{p}\left(\mathbf{u}^{*}(\lambda), \mathbf{v}\right)+a_{p}\left(\mathbf{u}^{*}(\mu), \mathbf{v}\right) \\
& =\left\langle\mathbf{v} \cdot \mathbf{n}_{p},(\lambda-\mu)\right\rangle_{\Gamma_{I}} .
\end{aligned}
$$

Thus, (with the assumption that meas $\left(\Gamma_{p, D}\right)>0$ ) employing a result analogous to Lemma 2 for $X_{p}$ and $Z_{p}$, we have

$$
\begin{aligned}
\|\lambda-\mu\|_{H^{\frac{1}{2}\left(\Gamma_{I}\right)}} & =\sup _{q \in H^{-\frac{1}{2}}\left(\Gamma_{I}\right)} \frac{\langle q,(\lambda-\mu)\rangle_{\Gamma_{I}}}{\|q\|_{H^{-\frac{1}{2}}\left(\Gamma_{I}\right)}} \\
& \leq C \sup _{\mathbf{v} \in \mathbf{X}_{p}} \frac{\left\langle\mathbf{v} \cdot \mathbf{n}_{p},(\lambda-\mu)\right\rangle_{\Gamma_{I}}}{\|\mathbf{v} \cdot \mathbf{n}\|_{H^{-\frac{1}{2}}}\left(\partial \Omega_{p}\right)} \\
& \leq C \sup _{\mathbf{v} \in \mathbf{Z}_{p}} \frac{\left\langle\mathbf{v} \cdot \mathbf{n}_{p},(\lambda-\mu)\right\rangle_{\Gamma_{I}}}{\|\mathbf{v} \cdot \mathbf{n}\|_{H^{-\frac{1}{2}}}\left(\Gamma_{I}\right)} \\
& \leq C\left\|\mathbf{u}^{*}(\lambda)-\mathbf{u}^{*}(\mu)\right\|_{\mathbf{X}_{p}} .
\end{aligned}
$$

Similar to the proof for Theorem 2, (3.45) and the second inequality in (3.46) can be shown by using the weak formulation (3.37)-(3.38) and the strict monotonicity of $a_{p}(\cdot, \cdot)$. One difference here is that we need to use the fact that the Darcy velocity is divergence free.

If $p_{I}$ is an unknown but the normal component of Darcy velocity $q_{I} \in H^{\frac{1}{2}}\left(\Gamma_{I}\right)$ is given instead, we can solve for the pressure on $\Gamma_{I}$ by seeking $\lambda \in H^{\frac{1}{2}}\left(\Gamma_{I}\right)$ such that

$$
A_{p}(\lambda, \mu)=-\int_{\Gamma_{I}} q_{I} \mu d s, \quad \forall \mu \in H^{\frac{1}{2}}\left(\Gamma_{I}\right)
$$


with the form $A_{p}(\cdot, \cdot)$ defined by

$$
A_{p}(\lambda, \mu):=-\left\langle\mathbf{u}_{p}^{*}(\lambda) \cdot \mathbf{n}_{p}, \mu\right\rangle_{\Gamma_{I}} .
$$

We now prove a few properties of $A_{p}(\cdot, \cdot)$.

Theorem 7. $A_{p}(\lambda, \mu)$ is a nonlinear functional of $\lambda$ and a linear functional of $\mu . A_{p}(\cdot, \cdot)$ is continuous on $H^{\frac{1}{2}}\left(\Gamma_{I}\right) \times H^{\frac{1}{2}}\left(\Gamma_{I}\right)$. Moreover, $A_{p}(\cdot, \cdot)$ is monotone on $H^{\frac{1}{2}}\left(\Gamma_{I}\right) \times H^{\frac{1}{2}}\left(\Gamma_{I}\right)$. If meas $\left(\Gamma_{p, D}\right)>0$, $A_{p}(\cdot, \cdot)$ is strictly monotone on $H^{\frac{1}{2}}\left(\Gamma_{I}\right) \times H^{\frac{1}{2}}\left(\Gamma_{I}\right)$.

Remark. Unlike the space on $\Gamma_{I}$ for Stokes flow, $\lambda$ and $\mu$ here need to be defined in $H^{\frac{1}{2}}\left(\Gamma_{I}\right)$, not $H^{-\frac{1}{2}}\left(\Gamma_{I}\right)$.

Proof. The continuity follows from Theorem 6 . To see the strict monotonicity under the assumption of meas $\left(\Gamma_{p, D}\right)>0$, we note

$$
\begin{aligned}
A_{p}(\lambda, \lambda-\mu)-A_{p}(\mu, \lambda-\mu) & =a_{p}\left(\mathbf{u}^{*}(\lambda), \mathbf{u}^{*}(\lambda)-\mathbf{u}^{*}(\mu)\right)-a_{p}\left(\mathbf{u}^{*}(\mu), \mathbf{u}^{*}(\lambda)-\mathbf{u}^{*}(\mu)\right) \\
& \geq C\left\|\mathbf{u}^{*}(\lambda)-\mathbf{u}^{*}(\mu)\right\|_{\left(L^{2}\left(\Omega_{p}\right)\right)^{d}}^{2} \\
& =C\left\|\mathbf{u}^{*}(\lambda)-\mathbf{u}^{*}(\mu)\right\|_{\mathbf{H}\left(\operatorname{div}, \Omega_{p}\right)}^{2} \\
& \geq C\|\lambda-\mu\|_{H^{\frac{1}{2}\left(\Gamma_{I}\right)}}^{2}
\end{aligned}
$$

where we have used Theorem 6 for the last inequality above. The rest of this theorem follows similarly as that in Theorem 3 .

Theorem 8. If meas $\left(\Gamma_{p, D}\right)>0$, then there is a unique solution $\lambda \in H^{\frac{1}{2}}\left(\Gamma_{I}\right)$ to the reduced problem (3.47) for any given $q_{I} \in H^{-\frac{1}{2}}\left(\Gamma_{I}\right)$.

Proof. This theorem follows directly from the continuity and the strict monotonicity of the form $A_{p}(\cdot, \cdot)$.

\subsection{Coupled system}

For convenience, in the following analysis we assume that meas $\left(\Gamma_{p, D}\right)>0$, which as we will see, gives the uniqueness of the pressure solution. We will remark later on the modification of the analysis for the case of meas $\left(\Gamma_{f, N}\right)=\operatorname{meas}\left(\Gamma_{p, D}\right)=0$.

We couple the fluid flow through the two regions using continuity of the flux on $\Gamma_{I}$. We define

$$
A(\lambda, \mu):=A_{f}(\lambda, \mu)+A_{p}(\lambda, \mu) .
$$

From the continuity of the normal velocity

$$
\left.[\mathbf{u} \cdot \mathbf{n}]\right|_{\Gamma_{I}}=\left.\left(\mathbf{u}_{f} \cdot \mathbf{n}_{f}+\mathbf{u}_{p} \cdot \mathbf{n}_{p}\right)\right|_{\Gamma_{I}}=0
$$

we know that the coupled system can be formulated as: Determine $\lambda \in H^{\frac{1}{2}}\left(\Gamma_{I}\right)$ such that

$$
A(\lambda, \mu)=0, \quad \forall \mu \in H^{\frac{1}{2}}\left(\Gamma_{I}\right) .
$$

We now list a few properties of $A(\cdot, \cdot)$. 
Theorem 9. $A(\lambda, \mu)$ is a nonlinear functional of $\lambda$ and a linear functional of $\mu$. Under the assumption that meas $\left(\Gamma_{p, D}\right)>0, A(\cdot, \cdot)$ is continuous and strictly monotone on $H^{\frac{1}{2}}\left(\Gamma_{I}\right) \times H^{\frac{1}{2}}\left(\Gamma_{I}\right)$.

Proof. It is clear that $A(\lambda, \mu)$ is linear in $\mu$. Continuity follows from the continuity of its parts:

$$
\begin{aligned}
|A(\lambda, \mu)-A(\hat{\lambda}, \mu)| & \leq\left|A_{f}(\lambda, \mu)-A_{f}(\widehat{\lambda}, \mu)\right|+\left|A_{p}(\lambda, \mu)-A_{p}(\widehat{\lambda}, \mu)\right| \\
& \leq C\|\lambda-\widehat{\lambda}\|_{H^{-\frac{1}{2}\left(\Gamma_{I}\right)}}\|\mu\|_{H^{-\frac{1}{2}\left(\Gamma_{I}\right)}}+C\|\lambda-\widehat{\lambda}\|_{H^{\frac{1}{2}\left(\Gamma_{I}\right)}}\|\mu\|_{H^{\frac{1}{2}\left(\Gamma_{I}\right)}} \\
& \leq C\|\lambda-\hat{\lambda}\|_{H^{\frac{1}{2}\left(\Gamma_{I}\right)}}\|\mu\|_{H^{\frac{1}{2}\left(\Gamma_{I}\right)}},
\end{aligned}
$$

and

$$
\begin{aligned}
|A(\lambda, \mu)-A(\lambda, \widehat{\mu})| & \leq\left|A_{f}(\lambda, \mu)-A_{f}(\lambda, \widehat{\mu})\right|+\left|A_{p}(\lambda, \mu)-A_{p}(\lambda, \widehat{\mu})\right| \\
& \leq C\|\lambda\|_{H^{\frac{1}{2}\left(\Gamma_{I}\right)}}\|\mu-\widehat{\mu}\|_{H^{\frac{1}{2}}\left(\Gamma_{I}\right)} .
\end{aligned}
$$

The strict monotonicity also follows from the individual monotonicities. That is, $A_{f}(\lambda, \lambda-\mu)-$ $A_{f}(\mu, \lambda-\mu) \geq 0$ and $A_{p}(\lambda, \lambda-\mu)-A_{p}(\mu, \lambda-\mu) \geq C\|\lambda-\mu\|_{H^{\frac{1}{2}\left(\Gamma_{I}\right)}}^{2}$ together imply $A(\lambda, \lambda-\mu)-$ $A(\mu, \lambda-\mu) \geq C\|\lambda-\mu\|_{H^{\frac{1}{2}}\left(\Gamma_{I}\right)}^{2}$

Theorem 10. We assume that meas $\left(\Gamma_{p, D}\right)>0$. For any given $q_{I} \in H^{\frac{1}{2}}\left(\Gamma_{I}\right)$, there is a unique solution $\lambda \in H^{\frac{1}{2}}\left(\Gamma_{I}\right)$ to the reduced problem (3.48).

Proof. This theorem follows directly from the continuity and the strict monotonicity of the form $A(\cdot, \cdot)$.

Theorem 11. We assume that meas $\left(\Gamma_{p, D}\right)>0$. Let $p_{I}=\lambda$ be the solution to the reduced interface problem (3.48), and $\mathbf{u}_{f}^{*}\left(p_{I}\right), p_{f}^{*}\left(p_{I}\right), \beta_{f}^{*}\left(p_{I}\right), \mathbf{u}_{p}^{*}\left(p_{I}\right), p_{p}^{*}\left(p_{I}\right)$, and $\beta_{p}^{*}\left(p_{I}\right)$ be the subdomain solutions to (3.18)-(3.19), and (3.37)-(3.38). We have the following regularity result:

$$
\begin{aligned}
\left\|p_{I}\right\|_{H^{\frac{1}{2}}\left(\Gamma_{I}\right)}+\| \mathbf{u}_{f}^{*}\left(p_{I}\right) & \left\|_{\mathbf{X}_{f}}+\right\| \mathbf{u}_{p}^{*}\left(p_{I}\right)\left\|_{\mathbf{X}_{p}}+\right\| p^{*}\left(p_{I}\right) \|_{L^{2}(\Omega)}+\left|\beta_{f}^{*}\left(p_{I}\right)\right|+\left|\beta_{p}^{*}\left(p_{I}\right)\right| \\
\leq & C\left(\left\|\mathbf{f}_{f}\right\|_{\mathbf{X}_{f}^{*}}+\left\|\mathbf{f}_{p}\right\|_{\mathbf{X}_{p}^{*}}+\left\|\boldsymbol{\tau}_{B}\right\|_{\left(H^{-\frac{1}{2}}\left(\Gamma_{f, N}\right)\right)}+\left\|p_{B}\right\|_{H^{\frac{1}{2}}\left(\Gamma_{p, D}\right)}+\left|q_{f}\right|+\left|q_{p}\right|\right) .
\end{aligned}
$$

Proof. From the weak formulation (3.48), we have

$$
A\left(p_{I}, p_{I}\right)=0 .
$$

Using this together with the continuity and strict monotonicity of the form $A(\cdot, \cdot)$, we conclude

$$
\begin{aligned}
\left\|p_{I}\right\|_{H^{\frac{1}{2}\left(\Gamma_{I}\right)}}^{2}=\left\|p_{I}-0\right\|_{H^{\frac{1}{2}}\left(\Gamma_{I}\right)}^{2} & \leq C\left(A\left(p_{I}, p_{I}-0\right)-A\left(0, p_{I}-0\right)\right) \\
& =-C A\left(0, p_{I}\right) \\
& =-C\left\langle\mathbf{u}_{f}^{*}(0) \cdot \mathbf{n}_{f}, p_{I}\right\rangle_{\Gamma_{I}}-C\left\langle\mathbf{u}_{p}^{*}(0) \cdot \mathbf{n}_{p}, p_{I}\right\rangle_{\Gamma_{I}} \\
& \leq \epsilon\left\|p_{I}\right\|_{H^{\frac{1}{2}\left(\Gamma_{I}\right)}}^{2}+C\left\|\mathbf{u}_{f}^{*}(0) \cdot \mathbf{n}_{f}\right\|_{H^{\frac{1}{2}}\left(\Gamma_{I}\right)}^{2}+C\left\|\mathbf{u}_{p}^{*}(0) \cdot \mathbf{n}_{p}\right\|_{H^{-\frac{1}{2}}\left(\Gamma_{I}\right)}^{2} \\
& \leq \epsilon\left\|p_{I}\right\|_{H^{\frac{1}{2}\left(\Gamma_{I}\right)}}^{2}+C\left\|\mathbf{u}_{f}^{*}(0)\right\|_{\mathbf{X}_{f}}^{2}+C\left\|\mathbf{u}_{p}^{*}(0)\right\|_{\mathbf{X}_{p}}^{2} .
\end{aligned}
$$


Consequently, we have

$$
\left\|p_{I}\right\|_{H^{\frac{1}{2}}\left(\Gamma_{I}\right)} \leq C\left\|\mathbf{u}_{f}^{*}(0)\right\|_{\mathbf{X}_{f}}+C\left\|\mathbf{u}_{p}^{*}(0)\right\|_{\mathbf{X}_{p}} .
$$

Theorems 1 and 5 imply

$$
\left\|\mathbf{u}_{f}^{*}(0)\right\|_{\mathbf{X}_{f}} \leq C\left(\left\|\mathbf{f}_{f}\right\|_{\mathbf{X}_{f}^{*}}+\left\|\boldsymbol{\tau}_{B}\right\|_{\left(H^{-\frac{1}{2}}\left(\Gamma_{f, N}\right)\right)^{d}}+\left|q_{f}\right|\right)
$$

and

$$
\left\|\mathbf{u}_{p}^{*}(0)\right\|_{\mathbf{X}_{p}} \leq C\left(\left\|\mathbf{f}_{p}\right\|_{\mathbf{X}_{p}^{*}}+\left\|p_{B}\right\|_{H^{\frac{1}{2}}\left(\Gamma_{p, D}\right)}+\left|q_{p}\right|\right) .
$$

This Theorem follows by another application of Theorems 1 and 5 .

Remark. If meas $\left(\Gamma_{f, N}\right)=\operatorname{meas}\left(\Gamma_{p, D}\right)=0$, the pressure solutions in the two stand-alone subdomain problems are still unique. But the pressure solution to the interface problem (3.48) is no longer unique; instead it is unique up to an additive constant. As a result, the two subdomain pressure solutions to the coupled system are unique up to an additive constant. In particular, we see that $A_{f}(\cdot, \cdot)$ and $A_{p}(\cdot, \cdot)$, and consequently $A(\cdot, \cdot)$, lose their strict monotonicity, which leads to the nonuniqueness of solutions. To see that the pressure solution actually exists uniquely up to an additive constant under meas $\left(\Gamma_{f, N}\right)=\operatorname{meas}\left(\Gamma_{p, D}\right)=0$, we replace the original spaces for $p_{f}, p_{p}$ and $p_{I}$ by $L^{2}\left(\Omega_{f}\right) / \mathbb{R}, L^{2}\left(\Omega_{p}\right) / \mathbb{R}$, and $H^{\frac{1}{2}}\left(\Gamma_{I}\right) / \mathbb{R}$, respectively. With the new spaces, one can show that $A_{f}(\cdot, \cdot)$ and $A_{p}(\cdot, \cdot)$, and consequently $A(\cdot, \cdot)$, recover their strict monotonicity under meas $\left(\Gamma_{f, N}\right)=$ meas $\left(\Gamma_{p, D}\right)=0$. All other theorems above then follow under the modified pressure spaces.

\section{Finite Element Approximations}

We discretize the coupled nonlinear Stokes-Darcy system (3.48), (3.18)-(3.19), and (3.37)-(3.38) with finite element approximations. We use conforming approximating spaces:

$$
\mathbf{X}_{f, h} \subset \mathbf{X}_{f}, \quad M_{f, h} \subset L^{2}\left(\Omega_{f}\right), \mathbf{X}_{p, h} \subset \mathbf{X}_{p}, \quad M_{p, h} \subset L^{2}\left(\Omega_{p}\right), \quad L_{h} \subset H^{\frac{1}{2}}\left(\Gamma_{I}\right) .
$$

Here $\mathbf{X}_{f, h}$ and $M_{f, h}$ denote velocity and pressure spaces typically used for Stokes fluid flow approximations, for example, the Taylor-Hood spaces. $\mathbf{X}_{p, h}$ and $M_{p, h}$ denote typical velocity and pressure spaces in mixed finite element methods for Darcy flow, such as the Raviart-Thomas spaces. In this section, we again assume that meas $\left(\Gamma_{p, D}\right)>0$ for the uniqueness of the pressure solution. We first list a few assumptions on our finite element spaces.

Assumption 1. We assume that there exists an inf-sup condition for the Stokes flow approximation:

$$
\inf _{\beta_{f} \in \mathbb{R}^{K_{f}, q_{h} \in M_{f, h}}} \sup _{\mathbf{v}_{h} \in \mathbf{X}_{f, h}} \frac{b_{f}\left(\mathbf{v}_{h}, q_{h}\right)+b_{f, B}\left(\mathbf{v}_{h}, \beta_{f}\right)}{\left\|\mathbf{v}_{h}\right\|_{\mathbf{X}_{f}}\left(\left\|q_{h}\right\|_{L^{2}\left(\Omega_{f}\right)}+\left|\beta_{f}\right|\right)} \geq C>0 .
$$

Remark. The above condition holds for the Taylor-Hood spaces on a quasi-uniform mesh of triangles or tetrahedra (see [2, 11]). 
Assumption 2. We assume that there exists an inf-sup condition for the Darcy flow approximation:

$$
\inf _{\beta_{p} \in \mathbb{R}^{K_{p}, q_{h} \in M_{p, h}}} \sup _{\mathbf{v}_{h} \in \mathbf{X}_{p, h}} \frac{b_{p}\left(\mathbf{v}_{h}, q_{h}\right)+b_{p, B}\left(\mathbf{v}_{h}, \beta_{p}\right)}{\left\|\mathbf{v}_{h}\right\|_{\mathbf{X}_{p}}\left(\left\|q_{h}\right\|_{L^{2}\left(\Omega_{p}\right)}+\left|\beta_{p}\right|\right)} \geq C>0 .
$$

Remark. The above assumption holds for the Raviart-Thomas spaces on a quasi-uniform mesh of triangles or tetrahedra (see [2, 11]).

Assumption 3. (Compatibility condition of the mixed spaces for Darcy flow) We assume that there exists a "swapped" inf-sup condition for the Darcy flow approximation:

$$
\inf _{\mathbf{v}_{h} \in \mathbf{X}_{p, h}} \sup _{q_{h} \in M_{p, h}} \frac{b_{p}\left(\mathbf{v}_{h}, q_{h}\right)}{\left\|\nabla \cdot \mathbf{v}_{h}\right\|_{L^{2}\left(\Omega_{p}\right)}\left\|q_{h}\right\|_{L^{2}\left(\Omega_{p}\right)}} \geq C>0 .
$$

Remark. The above assumption holds whenever $\nabla \cdot \mathbf{X}_{p, h} \subset M_{p, h}$, which is satisfied by commonly used mixed finite element spaces (for example, the Raviart-Thomas spaces).

Analogous to the analysis for the continuous problems, we define two null spaces:

$$
\begin{aligned}
& \mathbf{Z}_{f, h}:=\left\{\mathbf{v}_{h} \in \mathbf{X}_{f, h}: b_{f}\left(\mathbf{v}_{h}, q_{h}\right)+b_{f, B}\left(\mathbf{v}_{h}, \gamma_{f}\right)=0, \forall \gamma_{f} \in \mathbb{R}^{K_{f}}, \forall q_{h} \in M_{f, h}\right\}, \\
& \mathbf{Z}_{p, h}:=\left\{\mathbf{v}_{h} \in \mathbf{X}_{p, h}: b_{p}\left(\mathbf{v}_{h}, q_{h}\right)+b_{p, B}\left(\mathbf{v}_{h}, \gamma_{p}\right)=0, \forall \gamma_{p} \in \mathbb{R}^{K_{p}}, \forall q_{h} \in M_{p, h}\right\},
\end{aligned}
$$

and introduce two affine sets:

$$
\begin{aligned}
& \mathbf{Z}_{f, h}^{A}:=\left\{\mathbf{v}_{h} \in \mathbf{X}_{f, h}: b_{f}\left(\mathbf{v}_{h}, q_{h}\right)+b_{f, B}\left(\mathbf{v}_{h}, \gamma_{f}\right)=b_{f, B}\left(q_{f} \mathbf{n}_{f}, \gamma_{f}\right), \forall \gamma_{f} \in \mathbb{R}^{K_{f}}, \forall q_{h} \in M_{f, h}\right\}, \\
& \mathbf{Z}_{p, h}^{A}:=\left\{\mathbf{v}_{h} \in \mathbf{X}_{p, h}: b_{p}\left(\mathbf{v}_{h}, q_{h}\right)+b_{p, B}\left(\mathbf{v}_{h}, \gamma_{p}\right)=b_{f, B}\left(q_{p} \mathbf{n}_{p}, \gamma_{p}\right), \forall \gamma_{p} \in \mathbb{R}^{K_{p}}, \forall q_{h} \in M_{p, h}\right\} .
\end{aligned}
$$

Assumption 4. (Mortar compatibility condition for Stokes flow) We assume that there exists an inf-sup condition between the mortar space and the Stokes flow approximation spaces:

$$
\inf _{\lambda_{h} \in L_{h}} \sup _{\mathbf{v}_{h} \in \mathbf{Z}_{f, h}} \frac{\left\langle\mathbf{v}_{h} \cdot \mathbf{n}_{f}, \lambda_{h}\right\rangle_{\Gamma_{I}}}{\left\|\mathbf{v}_{h}\right\|_{\mathbf{X}_{f}}\left\|\lambda_{h}\right\|_{H^{-\frac{1}{2}}\left(\Gamma_{I}\right)}} \geq C .
$$

Assumption 5. (Mortar compatibility condition for Darcy flow) We assume that there exists an inf-sup condition between the mortar space and the Darcy flow approximation spaces:

$$
\inf _{\lambda_{h} \in L_{h}} \sup _{\mathbf{v}_{h} \in \mathbf{Z}_{p, h}} \frac{\left\langle\mathbf{v}_{h} \cdot \mathbf{n}_{p}, \lambda_{h}\right\rangle_{\Gamma_{I}}}{\left\|\mathbf{v}_{h}\right\|_{\mathbf{X}_{p}}\left\|\lambda_{h}\right\|_{H^{\frac{1}{2}}\left(\Gamma_{I}\right)}} \geq C .
$$

Remark. Roughly speaking, (4.52) specifies that the normal-velocity component restriction of $\mathbf{Z}_{f, h}$ on $\Gamma_{I}$ should be at least as dense as the mortar space $L_{h}$. Similarly, (4.53) specifies that the normalvelocity component restriction of $\mathbf{Z}_{p, h}$ on $\Gamma_{I}$ should be at least as dense as the mortar space $L_{h}$. We remark that it is sufficient but not necessary to require both mortar compatibility conditions (4.52) and (4.53) for the existence and uniqueness of a solution to the coupled system; either one of the two conditions is sufficient. We assume both compatibility conditions here for convenience.

Following from the above inf-sup assumptions and Theorem 18, we have the combined compatibility conditions: 
Lemma 4. There exist a constant $C>0$ such that

$$
\begin{aligned}
& \inf _{\lambda_{h} \in L_{h}, q_{h} \in M_{f, h}, \beta_{f} \in \mathbb{R}^{K}} \sup _{\mathbf{v}_{h} \in \mathbf{X}_{f, h}} \frac{\left\langle\lambda_{h}, \mathbf{v}_{h} \cdot \mathbf{n}_{f}\right\rangle_{\Gamma_{I}}+b_{f}\left(\mathbf{v}_{h}, q_{h}\right)+b_{f, B}\left(\mathbf{v}_{h}, \beta_{f}\right)}{\left\|\mathbf{v}_{h}\right\|_{\mathbf{X}_{f}}\left(\left\|\lambda_{h}\right\|_{H^{-\frac{1}{2}}\left(\Gamma_{I}\right)}+\left\|q_{h}\right\|_{L^{2}\left(\Omega_{f}\right)}+\left|\beta_{f}\right|\right)} \geq C>0, \\
& \inf _{\lambda_{h} \in L_{h}, q_{h} \in M_{p, h}, \beta_{p} \in \mathbb{R}^{K_{p}}} \sup _{\mathbf{v}_{h} \in \mathbf{X}_{p, h}} \frac{\left\langle\mathbf{v}_{h} \cdot \mathbf{n}_{p}, \lambda_{h}\right\rangle_{\Gamma_{I}}+b_{p}\left(\mathbf{v}_{h}, q_{h}\right)+b_{p, B}\left(\mathbf{v}_{h}, \beta_{p}\right)}{\left\|\mathbf{v}_{h}\right\|_{\mathbf{X}_{p}}\left(\left\|\lambda_{h}\right\|_{H^{\frac{1}{2}\left(\Gamma_{I}\right)}}+\left\|q_{h}\right\|_{L^{2}\left(\Omega_{p}\right)}+\left|\beta_{p}\right|\right)} \geq C>0 .
\end{aligned}
$$

\subsection{Stokes flow approximation}

We first restrict out attention to the finite element discretization of Stokes flow, assuming that $p_{I, h} \in L_{h} \subset H^{\frac{1}{2}}\left(\Gamma_{I}\right) \subset H^{-\frac{1}{2}}\left(\Gamma_{I}\right)$ is given. Define

$$
l_{f, h}(\mathbf{v}):=\left(f_{i}, v_{i}\right)_{\Omega_{f}}+\int_{\Gamma_{f, N}} \boldsymbol{\tau}_{B, i} v_{i} d s-\int_{\Gamma_{I}} p_{I, h} v_{i} n_{i} d s .
$$

The weak formulation is: Given $\mathbf{f} \in \mathbf{X}_{f}^{*}, \boldsymbol{\tau}_{N} \in\left(H^{-\frac{1}{2}}\left(\Gamma_{f, N}\right)\right)^{d}, q_{f} \in \mathbb{R}^{K_{F}}$, and $p_{I, h} \in L_{h}$, determine $\left(\mathbf{u}_{h}, p_{h}, \beta_{f, h}\right) \in \mathbf{X}_{f, h} \times M_{f, h} \times \mathbb{R}^{K_{f}}$ such that

$$
\begin{aligned}
a_{f}\left(\mathbf{u}_{h}, \mathbf{v}_{h}\right)-b_{f}\left(\mathbf{v}_{h}, p_{h}\right)-b_{f, B}\left(\mathbf{v}_{h}, \beta_{f, h}\right)=l_{f, h}\left(\mathbf{v}_{h}\right), & \forall \mathbf{v}_{h} \in \mathbf{X}_{f, h}, \\
b_{f}\left(\mathbf{u}_{h}, q_{h}\right)+b_{f, B}\left(\mathbf{u}_{h}, \gamma_{f}\right)=b_{f, B}\left(q_{f} \mathbf{n}_{f}, \gamma_{f},\right), & \forall\left(q_{h}, \gamma_{f}\right) \in M_{f, h} \times \mathbb{R}^{K_{f}} .
\end{aligned}
$$

Theorem 12. There exists a unique solution $\left(\mathbf{u}_{h}, p_{h}, \beta_{f, h}\right) \in \mathbf{X}_{f, h} \times M_{f, h} \times \mathbb{R}^{K_{f}}$ satisfying (4.56)(4.57). In addition, there exists a constant $C>0$ such that

$$
\begin{aligned}
\left\|\mathbf{u}-\mathbf{u}_{h}\right\|_{\mathbf{X}_{f}} & +\left\|p-p_{h}\right\|_{L^{2}\left(\Omega_{f}\right)}+\left|\beta_{f}-\beta_{f, h}\right| \\
& \leq C\left(\inf _{\widehat{\mathbf{u}}_{h} \in \mathbf{X}_{f, h}}\left\|\mathbf{u}-\widehat{\mathbf{u}}_{h}\right\|_{\mathbf{X}_{f}}+\inf _{\widehat{p}_{h} \in M_{f, h}}\left\|p-\widehat{p}_{h}\right\|_{L^{2}\left(\Omega_{f}\right)}\right)+C\left\|p_{I, h}-p_{I}\right\|_{H^{-\frac{1}{2}}\left(\Gamma_{I}\right)} .
\end{aligned}
$$

Proof. The existence and uniqueness of the solution follows from the continuity and strict monotonicity of $a_{f}(\cdot, \cdot)$ on $\mathbf{Z}_{f, h} \times \mathbf{Z}_{f, h}$, together with the discrete inf-sup condition (4.49).

Subtracting (3.18)-(3.19) from (4.56)-(4.57), respectively, we obtain the following error equations:

$$
\begin{aligned}
& a_{f}\left(\mathbf{u}_{h}, \mathbf{v}_{h}\right)=a_{f}\left(\mathbf{u}, \mathbf{v}_{h}\right)+b_{f}\left(\mathbf{v}_{h}, p_{h}-p\right)+b_{f, B}\left(\mathbf{v}_{h}, \beta_{f, h}-\beta_{f}\right) \\
& +l_{f, h}\left(\mathbf{v}_{h}\right)-l_{f}\left(\mathbf{v}_{h}\right), \quad \forall \mathbf{v}_{h} \in \mathbf{X}_{f, h}, \\
& b_{f}\left(\mathbf{u}_{h}-\mathbf{u}, q_{h}\right)+b_{f, B}\left(\mathbf{u}_{h}-\mathbf{u}, \gamma_{f}\right)=0, \quad \forall\left(q_{h}, \gamma_{f}\right) \in M_{f, h} \times \mathbb{R}^{K_{f}} .
\end{aligned}
$$

We note that the right-hand side of (4.59) contains the error propagated from the interface approximation:

$$
l_{f, h}\left(\mathbf{v}_{h}\right)-l_{f}\left(\mathbf{v}_{h}\right)=-\left\langle\left(p_{I, h}-p_{I}\right), \mathbf{v}_{h} \cdot \mathbf{n}\right\rangle_{\Gamma_{I}} .
$$

We pick $\widehat{\mathbf{u}}_{h} \in \mathbf{Z}_{f, h}^{A} \subset \mathbf{X}_{f, h}, \widehat{p}_{h} \in M_{f, h}, \widehat{\beta}_{f, h} \in \mathbb{R}^{K_{f}}$, and note that

$$
b_{f}\left(\mathbf{u}_{h}-\widehat{\mathbf{u}}_{h}, \widehat{p}_{h}-p_{h}\right)+b_{f, B}\left(\mathbf{u}_{h}-\widehat{\mathbf{u}}_{h}, \widehat{\beta}_{f, h}-\beta_{f, h}\right)=0 .
$$


With $\mathbf{v}_{h}=\mathbf{u}_{h}-\widehat{\mathbf{u}}_{h} \in \mathbf{Z}_{f, h} \subset \mathbf{X}_{f, h}$, using (4.59), (4.61) and (4.62), we obtain

$$
\begin{aligned}
a_{f}\left(\mathbf{u}_{h}, \mathbf{u}_{h}-\widehat{\mathbf{u}}_{h}\right)-a_{f}\left(\widehat{\mathbf{u}}_{h}, \mathbf{u}_{h}-\widehat{\mathbf{u}}_{h}\right)= & a_{f}\left(\mathbf{u}, \mathbf{u}_{h}-\widehat{\mathbf{u}}_{h}\right)-a_{f}\left(\widehat{\mathbf{u}}_{h}, \mathbf{u}_{h}-\widehat{\mathbf{u}}_{h}\right)+b_{f}\left(\mathbf{u}_{h}-\widehat{\mathbf{u}}_{h}, \widehat{p}_{h}-p\right) \\
& +b_{f, B}\left(\mathbf{u}_{h}-\widehat{\mathbf{u}}_{h}, \widehat{\beta}_{f, h}-\beta_{f}\right)-\left\langle\left(p_{I, h}-p_{I}\right),\left(\mathbf{u}_{h}-\widehat{\mathbf{u}}_{h}\right) \cdot \mathbf{n}\right\rangle_{\Gamma_{I}} \\
\leq & \epsilon\left\|\mathbf{u}_{h}-\widehat{\mathbf{u}}_{h}\right\|_{\mathbf{X}_{f}}^{2}+C\left\|\mathbf{u}-\widehat{\mathbf{u}}_{h}\right\|_{\mathbf{X}_{f}}^{2}+C\left\|p-\widehat{p}_{h}\right\|_{L^{2}\left(\Omega_{f}\right)}^{2} \\
& +C\left|\beta_{f}-\widehat{\beta}_{f, h}\right|^{2}+C\left\|p_{I, h}-p_{I}\right\|_{H^{-\frac{1}{2}}\left(\Gamma_{I}\right)}^{2} .
\end{aligned}
$$

Because of the strict monotonicity, we have

$$
a_{f}\left(\mathbf{u}_{h}, \mathbf{u}_{h}-\widehat{\mathbf{u}}_{h}\right)-a_{f}\left(\widehat{\mathbf{u}}_{h}, \mathbf{u}_{h}-\widehat{\mathbf{u}}_{h}\right) \geq C\left\|\mathbf{u}_{h}-\widehat{\mathbf{u}}_{h}\right\|_{\mathbf{X}_{f}}^{2} .
$$

Since $\widehat{\mathbf{u}}_{h}, \widehat{p}_{h}$, and $\widehat{\beta}_{f, h}$ can be arbitrarily chosen from their corresponding spaces, we have

$$
\begin{aligned}
\left\|\mathbf{u}_{h}-\widehat{\mathbf{u}}_{h}\right\|_{\mathbf{X}_{f}} \leq C\left(\inf _{\widehat{\mathbf{u}}_{h} \in \mathbf{Z}_{f, h}^{A}}\left\|\mathbf{u}-\widehat{\mathbf{u}}_{h}\right\|_{\mathbf{X}_{f}}+\inf _{\widehat{p}_{h} \in M_{f, h}}\left\|p-\widehat{p}_{h}\right\|_{L^{2}\left(\Omega_{f}\right)}+\inf _{\widehat{\beta}_{f, h} \in \mathbb{R}^{K_{f}}}\left|\beta_{f}-\widehat{\beta}_{f, h}\right|\right) \\
+C\left\|p_{I, h}-p_{I}\right\|_{H^{-\frac{1}{2}}\left(\Gamma_{I}\right)} \\
=C\left(\inf _{\widehat{\mathbf{u}}_{h} \in \mathbf{Z}_{f, h}^{A}}\left\|\mathbf{u}-\widehat{\mathbf{u}}_{h}\right\|_{\mathbf{X}_{f}}+\inf _{\widehat{p}_{h} \in M_{f, h}}\left\|p-\widehat{p}_{h}\right\|_{L^{2}\left(\Omega_{f}\right)}\right)+C\left\|p_{I, h}-p_{I}\right\|_{H^{-\frac{1}{2}}\left(\Gamma_{I}\right)} .
\end{aligned}
$$

We proceed to lift $\widehat{\mathbf{u}}_{h}$ from $\mathbf{Z}_{f, h}^{A}$ to $\mathbf{X}_{f, h}$ in the above estimate. From the inf-sup condition (4.49), we know that there exists an operator $\Pi_{h}: \mathbf{X}_{f} \rightarrow \mathbf{X}_{f, h}$ such that

$$
b_{f}\left(\mathbf{u}-\Pi_{h} \mathbf{u}, q_{h}\right)+b_{f, B}\left(\mathbf{u}-\Pi_{h} \mathbf{u}, \gamma_{f}\right)=0, \quad \forall\left(q_{h}, \gamma_{f}\right) \in M_{f, h} \times \mathbb{R}^{K_{f}}
$$

and

$$
\left\|\Pi_{h} \mathbf{u}\right\|_{\mathbf{X}_{f}} \leq C\|\mathbf{u}\|_{\mathbf{X}_{f}} .
$$

For any given $\widetilde{\mathbf{u}}_{h} \in \mathbf{X}_{f, h}$, we now define $\widehat{\mathbf{u}}_{h}:=\widetilde{\mathbf{u}}_{h}-\Pi_{h}\left(\widetilde{\mathbf{u}}_{h}-\mathbf{u}\right)$. Using (4.60) and that fact that $\mathbf{u}$ satisfies (3.19), we easily verify that $\widehat{\mathbf{u}}_{h} \in \mathbf{Z}_{f, h}^{A}$. We then have

$$
\begin{aligned}
\left\|\mathbf{u}-\widehat{\mathbf{u}}_{h}\right\|_{\mathbf{X}_{f}} & \leq\left\|\mathbf{u}-\widetilde{\mathbf{u}}_{h}\right\|_{\mathbf{X}_{f}}+\left\|\widetilde{\mathbf{u}}_{h}-\widehat{\mathbf{u}}_{h}\right\|_{\mathbf{X}_{f}} \\
& =\left\|\mathbf{u}-\widetilde{\mathbf{u}}_{h}\right\|_{\mathbf{X}_{f}}+\left\|\Pi_{h}\left(\widetilde{\mathbf{u}}_{h}-\mathbf{u}\right)\right\|_{\mathbf{X}_{f}} \\
& \leq(1+C)\left\|\mathbf{u}-\widetilde{\mathbf{u}}_{h}\right\|_{\mathbf{X}_{f}},
\end{aligned}
$$

which implies

$$
\inf _{\widehat{\mathbf{u}}_{h} \in \mathbf{Z}_{f, h}^{A}}\left\|\mathbf{u}-\widehat{\mathbf{u}}_{h}\right\|_{\mathbf{X}_{f}} \leq(1+C) \inf _{\widetilde{\mathbf{u}}_{h} \in \mathbf{X}_{f, h}}\left\|\mathbf{u}-\widetilde{\mathbf{u}}_{h}\right\|_{\mathbf{X}_{f}} .
$$

The triangle inequality together with (4.63) and (4.64) yield

$$
\left\|\mathbf{u}-\mathbf{u}_{h}\right\|_{\mathbf{X}_{f}} \leq C\left(\inf _{\widehat{\mathbf{u}}_{h} \in \mathbf{X}_{f, h}}\left\|\mathbf{u}-\widehat{\mathbf{u}}_{h}\right\|_{\mathbf{X}_{f}}+\inf _{\widehat{p}_{h} \in M_{f, h}}\left\|p-\widehat{p}_{h}\right\|_{L^{2}\left(\Omega_{f}\right)}\right)+C\left\|p_{I, h}-p_{I}\right\|_{H^{-\frac{1}{2}}\left(\Gamma_{I}\right)} .
$$


To get the error estimate for $p_{h}$ and $\beta_{f, h}$, we use the inf-sup condition (4.49) and the error equation (4.59). Picking $\widehat{p}_{h} \in M_{f, h}$ and $\widehat{\beta}_{f, h} \in \mathbb{R}^{K_{f}}$, we have

$$
\begin{aligned}
& \left\|p_{h}-\widehat{p}_{h}\right\|_{L^{2}\left(\Omega_{f}\right)}+\left|\beta_{f, h}-\widehat{\beta}_{f, h}\right| \leq C \sup _{\mathbf{v}_{h} \in \mathbf{X}_{f, h}} \frac{b_{f}\left(\mathbf{v}_{h}, p_{h}-\widehat{p}_{h}\right)+b_{f, B}\left(\mathbf{v}_{h}, \beta_{f, h}-\widehat{\beta}_{f, h}\right)}{\left\|\mathbf{v}_{h}\right\|_{\mathbf{X}_{f}}} \\
& \leq C \sup _{\mathbf{v}_{h} \in \mathbf{X}_{f, h}} \frac{b_{f}\left(\mathbf{v}_{h}, p_{h}-p\right)+b_{f, B}\left(\mathbf{v}_{h}, \beta_{f, h}-\beta_{f}\right)}{\left\|\mathbf{v}_{h}\right\|_{\mathbf{X}_{f}}} \\
& +C \sup _{\mathbf{v}_{h} \in \mathbf{X}_{f, h}} \frac{b_{f}\left(\mathbf{v}_{h}, p-\widehat{p}_{h}\right)+b_{f, B}\left(\mathbf{v}_{h}, \beta_{f}-\widehat{\beta}_{f, h}\right)}{\left\|\mathbf{v}_{h}\right\|_{\mathbf{X}_{f}}} \\
& =C \sup _{\mathbf{v}_{h} \in \mathbf{X}_{f, h}} \frac{a_{f}\left(\mathbf{u}_{h}, \mathbf{v}_{h}\right)-a_{f}\left(\mathbf{u}, \mathbf{v}_{h}\right)+\left\langle\left(p_{I, h}-p_{I}\right), \mathbf{v}_{h} \cdot \mathbf{n}\right\rangle_{\Gamma_{I}}}{\left\|\mathbf{v}_{h}\right\|_{\mathbf{X}_{f}}} \\
& +C \sup _{\mathbf{v}_{h} \in \mathbf{X}_{f, h}} \frac{b_{f}\left(\mathbf{v}_{h}, p-\widehat{p}_{h}\right)+b_{f, B}\left(\mathbf{v}_{h}, \beta_{f}-\widehat{\beta}_{f, h}\right)}{\left\|\mathbf{v}_{h}\right\|_{\mathbf{X}_{f}}} \\
& \leq C\left\|\mathbf{u}-\mathbf{u}_{h}\right\|_{\mathbf{X}_{f}}+C\left\|p_{I, h}-p_{I}\right\|_{H^{-\frac{1}{2}\left(\Gamma_{I}\right)}}+C\left\|p-\widehat{p}_{h}\right\|_{L^{2}\left(\Omega_{f}\right)}+C\left|\beta_{f}-\widehat{\beta}_{f, h}\right|,
\end{aligned}
$$

implying

$$
\begin{aligned}
\left\|p-p_{h}\right\|_{L^{2}\left(\Omega_{p}\right)}+\left|\beta_{f}-\beta_{f, h}\right| \leq & C\left\|\mathbf{u}-\mathbf{u}_{h}\right\|_{\mathbf{X}_{f}}+C \inf _{\widehat{p}_{h} \in M_{f, h}}\left\|p-\widehat{p}_{h}\right\|_{L^{2}\left(\Omega_{f}\right)} \\
& +C \inf _{\widehat{\beta}_{f, h} \in \mathbb{R}^{K_{f}}}\left|\beta_{f}-\widehat{\beta}_{f, h}\right|+C\left\|p_{I, h}-p_{I}\right\|_{H^{-\frac{1}{2}}\left(\Gamma_{I}\right)} \\
= & C\left\|\mathbf{u}-\mathbf{u}_{h}\right\|_{\mathbf{X}_{f}}+C \inf _{\widehat{p}_{h} \in M_{f, h}}\left\|p-\widehat{p}_{h}\right\|_{L^{2}\left(\Omega_{f}\right)}+C\left\|p_{I, h}-p_{I}\right\|_{H^{-\frac{1}{2}\left(\Gamma_{I}\right)}} .
\end{aligned}
$$

The estimate (4.58) follows from (4.65) and (4.66).

\subsection{Darcy flow approximation}

We now consider the finite element discretization for the Darcy flow, assuming that $p_{I, h} \in L_{h} \subset$ $H^{\frac{1}{2}}\left(\Gamma_{I}\right)$ is given. We define

$$
l_{p, h}(\mathbf{v}):=\left(f_{i}, v_{i}\right)_{\Omega_{p}}-\left\langle\mathbf{v}_{h} \cdot \mathbf{n}_{p}, p_{B}\right\rangle_{\Gamma_{p, D}}-\left\langle\mathbf{v}_{h} \cdot \mathbf{n}_{p}, p_{I, h}\right\rangle_{\Gamma_{I}}
$$

The weak formulation is: Given $\mathbf{f} \in \mathbf{X}_{p}^{*}, p_{B} \in H^{\frac{1}{2}}\left(\Gamma_{p, D}\right), q_{p} \in \mathbb{R}^{K_{p}}$, and $p_{I, h} \in L_{h}$, determine $\left(\mathbf{u}_{h}, p_{h}, \beta_{p, h}\right) \in \mathbf{X}_{p, h} \times M_{p, h} \times \mathbb{R}^{K_{p}}$ such that

$$
\begin{aligned}
a_{p}\left(\mathbf{u}_{h}, \mathbf{v}_{h}\right)-b_{p}\left(\mathbf{v}_{h}, p_{h}\right)-b_{p, B}\left(\mathbf{v}_{h}, \beta_{p, h}\right) & =l_{p, h}\left(\mathbf{v}_{h}\right), & \forall \mathbf{v}_{h} \in \mathbf{X}_{p, h}, \\
b_{p}\left(\mathbf{u}_{h}, q_{h}\right)+b_{p, B}\left(\mathbf{u}_{h}, \gamma_{p}\right) & =b_{p, B}\left(q_{p} \mathbf{n}_{p}, \gamma_{p}\right), & \forall\left(q_{h}, \gamma_{p}\right) \in M_{p, h} \times \mathbb{R}^{K_{p}} .
\end{aligned}
$$


Theorem 13. There exists a unique solution $\left(\mathbf{u}_{h}, p_{h}, \beta_{p, h}\right) \in \mathbf{X}_{p, h} \times M_{p, h} \times \mathbb{R}^{K_{p}}$ satisfying (4.67)(4.68). In addition, there exists a constant $C>0$ such that

$$
\begin{aligned}
\left\|\mathbf{u}-\mathbf{u}_{h}\right\|_{\mathbf{X}_{p}} & +\left\|p-p_{h}\right\|_{L^{2}\left(\Omega_{p}\right)}+\left|\beta_{p}-\beta_{p, h}\right| \\
& \leq C\left(\inf _{\widehat{\mathbf{u}}_{h} \in \mathbf{X}_{p, h}}\left\|\mathbf{u}-\widehat{\mathbf{u}}_{h}\right\|_{\mathbf{X}_{p}}+\inf _{\widehat{p}_{h} \in M_{p, h}}\left\|p-\widehat{p}_{h}\right\|_{L^{2}\left(\Omega_{p}\right)}\right)+C\left\|p_{I, h}-p_{I}\right\|_{H^{\frac{1}{2}\left(\Gamma_{I}\right)}} .
\end{aligned}
$$

Proof. The existence and uniqueness of the solution follows from the continuity and strict monotonicity of $a_{p}(\cdot, \cdot)$ on $\mathbf{Z}_{p, h} \times \mathbf{Z}_{p, h}$, together with the discrete inf-sup condition (4.50).

Subtracting (3.37)-(3.38) from (4.67)-(4.68), respectively, we obtain the error equations:

$$
\begin{gathered}
a_{p}\left(\mathbf{u}_{h}, \mathbf{v}_{h}\right)-a_{p}\left(\mathbf{u}, \mathbf{v}_{h}\right)-b_{p}\left(\mathbf{v}_{h}, p_{h}-p\right)-b_{p, B}\left(\mathbf{v}_{h}, \beta_{p, h}-\beta_{p}\right) \\
=-\left\langle\mathbf{v}_{h} \cdot \mathbf{n}_{p},\left(p_{I, h}-p_{I}\right)\right\rangle_{\Gamma_{I}}, \quad \forall \mathbf{v}_{h} \in \mathbf{X}_{p, h}, \\
b_{p}\left(\mathbf{u}_{h}-\mathbf{u}, q_{h}\right)+b_{p, B}\left(\mathbf{u}_{h}-\mathbf{u}, \gamma_{p}\right)=0, \quad \forall\left(q_{h}, \gamma_{p}\right) \in M_{p, h} \times \mathbb{R}^{K_{p}} .
\end{gathered}
$$

We now take $\widehat{\mathbf{u}}_{h} \in \mathbf{Z}_{p, h}^{A} \subset \mathbf{X}_{p, h}, \widehat{p}_{h} \in M_{p, h}, \widehat{\beta}_{p, h} \in \mathbb{R}^{K_{p}}$, and set $\mathbf{v}_{h}=\mathbf{u}_{h}-\widehat{\mathbf{u}}_{h} \in \mathbf{Z}_{p, h} \subset \mathbf{X}_{p, h}$ in (4.70) to obtain

$$
\begin{aligned}
a_{p}\left(\mathbf{u}_{h}, \mathbf{u}_{h}-\widehat{\mathbf{u}}_{h}\right)-a_{p}\left(\widehat{\mathbf{u}}_{h}, \mathbf{u}_{h}-\widehat{\mathbf{u}}_{h}\right)= & a_{p}\left(\mathbf{u}, \mathbf{u}_{h}-\widehat{\mathbf{u}}_{h}\right)-a_{p}\left(\widehat{\mathbf{u}}_{h}, \mathbf{u}_{h}-\widehat{\mathbf{u}}_{h}\right)+b_{p}\left(\mathbf{u}_{h}-\widehat{\mathbf{u}}_{h}, \widehat{p}_{h}-p\right) \\
& +b_{p, B}\left(\mathbf{u}_{h}-\widehat{\mathbf{u}}_{h}, \widehat{\beta}_{p, h}-\beta_{p}\right)-\left\langle\left(\mathbf{u}_{h}-\widehat{\mathbf{u}}_{h}\right) \cdot \mathbf{n}_{p},\left(p_{I, h}-p_{I}\right)\right\rangle_{\Gamma_{I}} \\
\leq & \epsilon\left\|\mathbf{u}_{h}-\widehat{\mathbf{u}}_{h}\right\|_{\mathbf{X}_{p}}^{2}+C\left\|\mathbf{u}-\widehat{\mathbf{u}}_{h}\right\|_{\left(L^{2}\left(\Omega_{p}\right)\right)^{d}}^{2}+C\left\|p-\widehat{p}_{h}\right\|_{L^{2}\left(\Omega_{p}\right)}^{2} \\
& +C\left|\beta_{p}-\widehat{\beta}_{p, h}\right|^{2}+C\left\|p_{I, h}-p_{I}\right\|_{H^{\frac{1}{2}\left(\Gamma_{I}\right)}}^{2} .
\end{aligned}
$$

Due to the strict monotonicity of $a_{p}(\cdot, \cdot)$, we have

$$
a_{p}\left(\mathbf{u}_{h}, \mathbf{u}_{h}-\widehat{\mathbf{u}}_{h}\right)-a_{p}\left(\widehat{\mathbf{u}}_{h}, \mathbf{u}_{h}-\widehat{\mathbf{u}}_{h}\right) \geq C\left\|\mathbf{u}_{h}-\widehat{\mathbf{u}}_{h}\right\|_{\left(L^{2}\left(\Omega_{p}\right)\right)^{d}}^{2} .
$$

Since the $\widehat{\mathbf{u}}_{h}, \widehat{p}_{h}$, and $\widehat{\beta}_{p, h}$ can be arbitrarily chosen from their corresponding spaces, we have

$$
\begin{aligned}
\left\|\mathbf{u}-\mathbf{u}_{h}\right\|_{\left(L^{2}\left(\Omega_{p}\right)\right)^{d}} \leq & \inf _{\widehat{\mathbf{u}}_{h} \in \mathbf{Z}_{p, h}^{A}}\left(\left\|\mathbf{u}-\widehat{\mathbf{u}}_{h}\right\|_{\left(L^{2}\left(\Omega_{p}\right)\right)^{d}}+\left\|\widehat{\mathbf{u}}_{h}-\mathbf{u}_{h}\right\|_{\left(L^{2}\left(\Omega_{p}\right)\right)^{d}}\right) \\
\leq & C\left(\inf _{\widehat{\mathbf{u}}_{h} \in \mathbf{Z}_{p, h}^{A}}\left\|\mathbf{u}-\widehat{\mathbf{u}}_{h}\right\|_{\left(L^{2}\left(\Omega_{p}\right)\right)^{d}}+\inf _{\widehat{p}_{h} \in M_{p, h}}\left\|p-\widehat{p}_{h}\right\|_{L^{2}\left(\Omega_{p}\right)}+\inf _{\widehat{\beta}_{p, h} \in \mathbb{R}^{K_{p}}}\left|\beta_{p}-\widehat{\beta}_{p, h}\right|\right) \\
& +C\left\|p_{I, h}-p_{I}\right\|_{H^{\frac{1}{2}\left(\Gamma_{I}\right)}}+\epsilon \inf _{\widehat{\mathbf{u}}_{h} \in \mathbf{Z}_{p, h}^{A}}\left\|\mathbf{u}_{h}-\widehat{\mathbf{u}}_{h}\right\|_{\mathbf{X}_{p}} \\
\leq & C\left(\inf _{\widehat{\mathbf{u}}_{h} \in \mathbf{Z}_{p, h}^{A}}\left\|\mathbf{u}-\widehat{\mathbf{u}}_{h}\right\|_{\mathbf{X}_{p}}+\inf _{\widehat{p}_{h} \in M_{p, h}}\left\|p-\widehat{p}_{h}\right\|_{L^{2}\left(\Omega_{p}\right)}\right) \\
& +C\left\|p_{I, h}-p_{I}\right\|_{H^{\frac{1}{2}\left(\Gamma_{I}\right)}}+\epsilon\left\|\mathbf{u}-\mathbf{u}_{h}\right\|_{\mathbf{X}_{p}} .
\end{aligned}
$$

Now we use the swapped inf-sup condition (4.51) and 4.68 to obtain

$$
\left\|\nabla \cdot \mathbf{u}_{h}\right\|_{L^{2}\left(\Omega_{p}\right)} \leq C \sup _{q_{h} \in M_{p, h}} \frac{b_{p}\left(\mathbf{u}_{h}, q_{h}\right)}{\left\|q_{h}\right\|_{L^{2}\left(\Omega_{p}\right)}}=0
$$


which then implies

$$
\left\|\nabla \cdot\left(\mathbf{u}_{h}-\mathbf{u}\right)\right\|_{L^{2}\left(\Omega_{p}\right)}=0 .
$$

As a consequence of (4.72) and (4.73), we have

$$
\left\|\mathbf{u}-\mathbf{u}_{h}\right\|_{\mathbf{X}_{p}} \leq C\left(\inf _{\widehat{\mathbf{u}}_{h} \in \mathbf{Z}_{p, h}^{A}}\left\|\mathbf{u}-\widehat{\mathbf{u}}_{h}\right\|_{\mathbf{X}_{p}}+\inf _{\widehat{p}_{h} \in M_{p, h}}\left\|p-\widehat{p}_{h}\right\|_{L^{2}\left(\Omega_{p}\right)}\right)+C\left\|p_{I, h}-p_{I}\right\|_{H^{\frac{1}{2}\left(\Gamma_{I}\right)}} .
$$

Using the inf-sup condition (4.50) and the fact that $\mathbf{u}$ satisfies (3.38), we now lift $\widehat{\mathbf{u}}_{h}$ from $\mathbf{Z}_{p, h}^{A}$ to $\mathbf{X}_{p, h}$ by applying an argument similar to the one we have used before for lifting the velocity in the Stokes flow region to obtain:

$$
\inf _{\widehat{\mathbf{u}}_{h} \in \mathbf{Z}_{p, h}^{A}}\left\|\mathbf{u}-\widehat{\mathbf{u}}_{h}\right\|_{\mathbf{X}_{p}} \leq C \inf _{\widetilde{\mathbf{u}}_{h} \in \mathbf{X}_{p, h}}\left\|\mathbf{u}-\widetilde{\mathbf{u}}_{h}\right\|_{\mathbf{X}_{p}} .
$$

Now (4.74) and (4.75) yield

$$
\left\|\mathbf{u}-\mathbf{u}_{h}\right\|_{\mathbf{X}_{p}} \leq C\left(\inf _{\widehat{\mathbf{u}}_{h} \in \mathbf{X}_{p, h}}\left\|\mathbf{u}-\widehat{\mathbf{u}}_{h}\right\|_{\mathbf{X}_{p}}+\inf _{\widehat{p}_{h} \in M_{p, h}}\left\|p-\widehat{p}_{h}\right\|_{L^{2}\left(\Omega_{p}\right)}\right)+C\left\|p_{I, h}-p_{I}\right\|_{H^{\frac{1}{2}}\left(\Gamma_{I}\right)} .
$$

To get the error estimate for $p_{h}$ and $\beta_{p, h}$, we use the inf-sup condition (4.50) and the error equation (4.70) by picking $\widehat{p}_{h} \in M_{p, h}$ and $\widehat{\beta}_{p, h} \in \mathbb{R}^{K_{p}}$ similar to what we have done for Stokes flow:

$$
\begin{aligned}
\left\|p_{h}-\widehat{p}_{h}\right\|_{L^{2}\left(\Omega_{p}\right)}+\left|\beta_{p, h}-\widehat{\beta}_{p, h}\right| \leq & C \sup _{\mathbf{v}_{h} \in \mathbf{X}_{p, h}} \frac{b_{p}\left(\mathbf{v}_{h}, p_{h}-\widehat{p}_{h}\right)+b_{p, B}\left(\mathbf{v}_{h}, \beta_{p, h}-\widehat{\beta}_{p, h}\right)}{\left\|\mathbf{v}_{h}\right\|_{\mathbf{X}_{p}}} \\
\leq & C \sup _{\mathbf{v}_{h} \in \mathbf{X}_{p, h}} \frac{b_{p}\left(\mathbf{v}_{h}, p_{h}-p\right)+b_{p, B}\left(\mathbf{v}_{h}, \beta_{p, h}-\beta_{p}\right)}{\left\|\mathbf{v}_{h}\right\|_{\mathbf{X}_{p}}} \\
& +C \sup _{\mathbf{v}_{h} \in \mathbf{X}_{p, h}} \frac{b_{p}\left(\mathbf{v}_{h}, p-\widehat{p}_{h}\right)+b_{p, B}\left(\mathbf{v}_{h}, \beta_{p}-\widehat{\beta}_{p, h}\right)}{\left\|\mathbf{v}_{h}\right\|_{\mathbf{X}_{p}}} \\
= & C \sup _{\mathbf{v}_{h} \in \mathbf{X}_{p, h}} \frac{a_{p}\left(\mathbf{u}_{h}, \mathbf{v}_{h}\right)-a_{p}\left(\mathbf{u}, \mathbf{v}_{h}\right)+\left\langle\mathbf{v}_{h} \cdot \mathbf{n}_{p},\left(p_{I, h}-p_{I}\right)\right\rangle_{\Gamma_{I}}}{\left\|\mathbf{v}_{h}\right\|_{\mathbf{X}_{p}}} \\
& +C \sup _{\mathbf{v}_{h} \in \mathbf{X}_{p, h}} \frac{b_{p}\left(\mathbf{v}_{h}, p-\widehat{p}_{h}\right)+b_{p, B}\left(\mathbf{v}_{h}, \beta_{p}-\widehat{\beta}_{p, h}\right)}{\left\|\mathbf{v}_{h}\right\|_{\mathbf{X}_{p}}} \\
\leq & C \|_{\mathbf{u}-\mathbf{u}_{h}\left\|_{\mathbf{x}_{p}}+C\right\| p_{I, h}-p_{I}\left\|_{H^{\frac{1}{2}}\left(\Gamma_{I}\right)}+C\right\| p-\widehat{p}_{h} \|_{L^{2}\left(\Omega_{f}\right)}+C\left|\beta_{p}-\widehat{\beta}_{p, h}\right|,}
\end{aligned}
$$

implying

$$
\begin{aligned}
\left\|p-p_{h}\right\|_{L^{2}\left(\Omega_{p}\right)}+\left|\beta_{p}-\beta_{p, h}\right| \leq & C\left\|\mathbf{u}-\mathbf{u}_{h}\right\|_{\mathbf{X}_{p}}+C \inf _{\widehat{p}_{h} \in M_{p, h}}\left\|p-\widehat{p}_{h}\right\|_{L^{2}\left(\Omega_{p}\right)} \\
& +C \inf _{\widehat{\beta}_{p, h} \in \mathbb{R}^{K_{p}}}\left|\beta_{p}-\widehat{\beta}_{p, h}\right|+C\left\|p_{I, h}-p_{I}\right\|_{H^{\frac{1}{2}\left(\Gamma_{I}\right)}} \\
= & C\left\|\mathbf{u}-\mathbf{u}_{h}\right\|_{\mathbf{X}_{p}}+C \inf _{\widehat{p}_{h} \in M_{p, h}}\left\|p-\widehat{p}_{h}\right\|_{L^{2}\left(\Omega_{p}\right)}+C\left\|p_{I, h}-p_{I}\right\|_{H^{\frac{1}{2}\left(\Gamma_{I}\right)}} .
\end{aligned}
$$

The estimate (4.69) follows from (4.76) and (4.77). 


\subsection{Discretization on the interface}

For each $p_{I, h} \in L_{h} \subset H^{\frac{1}{2}}\left(\Gamma_{I}\right) \subset H^{-\frac{1}{2}}\left(\Gamma_{I}\right)$, we have a unique finite element solution $\left(\mathbf{u}_{f, h}^{*}\left(p_{I, h}\right), p_{f, h}^{*}\left(p_{I, h}\right), \beta_{f, h}^{*}\left(p_{I, h}\right)\right) \in \mathbf{X}_{f, h} \times M_{f, h} \times \mathbb{R}^{K_{f}}$ satisfying (4.56)-(4.57), and a unique finite element solution $\left(\mathbf{u}_{p, h}^{*}\left(p_{I, h}\right), p_{p, h}^{*}\left(p_{I, h}\right), \beta_{p, h}^{*}\left(p_{I, h}\right)\right) \in \mathbf{X}_{p, h} \times M_{p, h} \times \mathbb{R}^{K_{p}}$ satisfying (4.67)-(4.68). We now define

$$
\begin{aligned}
A_{f, h}\left(\lambda_{h}, \mu_{h}\right) & :=-\left\langle\mu_{h}, \mathbf{u}_{f, h}^{*}\left(\lambda_{h}\right) \cdot \mathbf{n}_{f}\right\rangle_{\Gamma_{I}}, \\
A_{p, h}\left(\lambda_{h}, \mu_{h}\right) & :=-\left\langle\mathbf{u}_{p, h}^{*}\left(\lambda_{h}\right) \cdot \mathbf{n}_{p}, \mu_{h}\right\rangle_{\Gamma_{I}}, \\
A_{h}\left(\lambda_{h}, \mu_{h}\right) & :=A_{f, h}\left(\lambda_{h}, \mu_{h}\right)+A_{p, h}\left(\lambda_{h}, \mu_{h}\right) .
\end{aligned}
$$

The discretized interface problem for the coupled system can be formulated as: Determine $\lambda_{h} \in$ $L_{h} \subset H^{\frac{1}{2}}\left(\Gamma_{I}\right)$ such that

$$
A_{h}\left(\lambda_{h}, \mu_{h}\right)=0, \quad \forall \mu \in L_{h} .
$$

Theorem 14. There exists a constant $C$ such that

$$
\begin{gathered}
\frac{1}{C}\left\|\lambda_{h}-\mu_{h}\right\|_{H^{-\frac{1}{2}\left(\Gamma_{I}\right)}} \leq\left\|\mathbf{u}_{f, h}^{*}\left(\lambda_{h}\right)-\mathbf{u}_{f, h}^{*}\left(\mu_{h}\right)\right\|_{\mathbf{X}_{f}} \leq C\left\|\lambda_{h}-\mu_{h}\right\|_{H^{-\frac{1}{2}\left(\Gamma_{I}\right)}}, \\
\frac{1}{C}\left\|\lambda_{h}-\mu_{h}\right\|_{H^{\frac{1}{2}\left(\Gamma_{I}\right)}} \leq\left\|\mathbf{u}_{p, h}^{*}\left(\lambda_{h}\right)-\mathbf{u}_{p, h}^{*}\left(\mu_{h}\right)\right\|_{\mathbf{X}_{p}} \leq C\left\|\lambda_{h}-\mu_{h}\right\|_{H^{\frac{1}{2}}\left(\Gamma_{I}\right)} .
\end{gathered}
$$

Proof. (4.79) and (4.80) can be shown similarly as we did for Theorems 2 and 6, except that we now need to replace the continuous inf-sup conditions by their discrete counterparts, i.e. the mortar compatibility conditions (4.52) and (4.53) for the lower bounds. We point out that the proof for the upper bound part of (4.80) uses the property that $\mathbf{u}_{h}$ is divergence free in the Darcy region.

We now prove a few properties of $A_{h}(\cdot, \cdot)$.

Theorem 15. $A_{h}\left(\lambda_{h}, \mu_{h}\right)$ is a nonlinear functional of $\lambda_{h}$ and a linear functional of $\mu_{h} . A_{h}(\cdot, \cdot)$ is continuous and strictly monotone on $L_{h} \times L_{h}$.

Proof. It is clear that $A_{h}(\lambda, \mu)$ is linear in $\mu$. To see the continuity, we apply Theorem 14:

$$
\begin{aligned}
\left|A_{h}\left(\lambda_{h}, \mu_{h}\right)-A_{h}\left(\widehat{\lambda}_{h}, \mu_{h}\right)\right| \leq & \left|A_{f, h}\left(\lambda_{h}, \mu_{h}\right)-A_{f, h}\left(\widehat{\lambda}_{h}, \mu_{h}\right)\right|+\left|A_{p, h}\left(\lambda_{h}, \mu_{h}\right)-A_{p, h}\left(\widehat{\lambda}_{h}, \mu_{h}\right)\right| \\
\leq & C\left\|\mathbf{u}_{f, h}^{*}\left(\lambda_{h}\right)-\mathbf{u}_{f, h}^{*}\left(\widehat{\lambda}_{h}\right)\right\|_{\mathbf{X}_{f}}\left\|\mu_{h}\right\|_{H^{-\frac{1}{2}}\left(\Gamma_{I}\right)} \\
& +C\left\|\mathbf{u}_{p, h}^{*}\left(\lambda_{h}\right)-\mathbf{u}_{p, h}^{*}\left(\widehat{\lambda}_{h}\right)\right\|_{\mathbf{X}_{p}}\left\|\mu_{h}\right\|_{H^{\frac{1}{2}}\left(\Gamma_{I}\right)} \\
\leq & C\left\|\lambda_{h}-\widehat{\lambda}_{h}\right\|_{H^{-\frac{1}{2}\left(\Gamma_{I}\right)}}\left\|\mu_{h}\right\|_{H^{-\frac{1}{2}\left(\Gamma_{I}\right)}}+C\left\|\lambda_{h}-\widehat{\lambda}_{h}\right\|_{H^{\frac{1}{2}\left(\Gamma_{I}\right)}}\left\|\mu_{h}\right\|_{H^{\frac{1}{2}}\left(\Gamma_{I}\right)} \\
\leq & C\left\|\lambda_{h}-\widehat{\lambda}_{h}\right\|_{H^{\frac{1}{2}\left(\Gamma_{I}\right)}}\left\|\mu_{h}\right\|_{H^{\frac{1}{2}\left(\Gamma_{I}\right)}},
\end{aligned}
$$


and

$$
\begin{aligned}
\left|A_{h}\left(\lambda_{h}, \mu_{h}\right)-A_{h}\left(\lambda_{h}, \widehat{\mu}_{h}\right)\right| & \leq\left|A_{f, h}\left(\lambda_{h}, \mu_{h}\right)-A_{f, h}\left(\lambda_{h}, \widehat{\mu}_{h}\right)\right|+\left|A_{p, h}\left(\lambda_{h}, \mu_{h}\right)-A_{p, h}\left(\lambda_{h}, \widehat{\mu}_{h}\right)\right| \\
& \leq C\left\|\lambda_{h}\right\|_{H^{\frac{1}{2}\left(\Gamma_{I}\right)}}\left\|\mu_{h}-\widehat{\mu}_{h}\right\|_{H^{\frac{1}{2}}\left(\Gamma_{I}\right)} .
\end{aligned}
$$

Like its continuous counterpart in Theorem 3, we have the following strict monotonicity

$$
\begin{aligned}
A_{f, h}\left(\lambda_{h}, \lambda_{h}-\mu_{h}\right)-A_{f, h}\left(\mu_{h}, \lambda_{h}-\mu_{h}\right)= & a_{f}\left(\mathbf{u}_{f, h}^{*}\left(\lambda_{h}\right), \mathbf{u}_{f, h}^{*}\left(\lambda_{h}\right)-\mathbf{u}_{f, h}^{*}\left(\mu_{h}\right)\right) \\
& -a_{f}\left(\mathbf{u}_{f, h}^{*}\left(\mu_{h}\right), \mathbf{u}_{f, h}^{*}\left(\lambda_{h}\right)-\mathbf{u}_{f, h}^{*}\left(\mu_{h}\right)\right) \\
\geq & C\left\|\mathbf{u}_{f, h}^{*}\left(\lambda_{h}\right)-\mathbf{u}_{f, h}^{*}\left(\mu_{h}\right)\right\|_{\mathbf{X}_{f}}^{2} \\
\geq & C\left\|\lambda_{h}-\mu_{h}\right\|_{H^{-\frac{1}{2}}\left(\Gamma_{I}\right)}^{2} .
\end{aligned}
$$

Similarly, we have

$$
A_{p, h}\left(\lambda_{h}, \lambda_{h}-\mu_{h}\right)-A_{p, h}\left(\mu_{h}, \lambda_{h}-\mu_{h}\right) \geq C\left\|\lambda_{h}-\mu_{h}\right\|_{H^{\frac{1}{2}\left(\Gamma_{I}\right)}}^{2} .
$$

The strict monotonicity of $A_{h}(\cdot, \cdot)$ then follows directly from these two inequalities.

Theorem 16. For any given $q_{I} \in H^{\frac{1}{2}}\left(\Gamma_{I}\right)$, there is a unique solution $\lambda_{h} \in L_{h}$ to the reduced problem (4.78).

Proof. This theorem directly follow from the continuity and the strict monotonicity of the form $A_{h}(\cdot, \cdot)$.

Theorem 17. Let $p_{I, h}=\lambda_{h}$ be the solution to the discretized interface problem (4.78). Let $\left(\mathbf{u}_{f, h}, p_{f, h}, \beta_{f, h}\right)$ and $\left(\mathbf{u}_{p, h}, p_{p, h}, \beta_{p, h}\right)$ be the subdomain solutions to (4.56)-(4.57) and (4.67)-(4.68) supplied with $p_{I, h}$. Then, there exists a constant $C$ such that

$$
\begin{aligned}
& \left\|p_{I}-p_{I, h}\right\|_{H^{\frac{1}{2}\left(\Gamma_{I}\right)}}+\left(\left\|\mathbf{u}_{f}-\mathbf{u}_{f, h}\right\|_{\mathbf{X}_{f}}+\left\|p_{f}-p_{f, h}\right\|_{L^{2}\left(\Omega_{f}\right)}+\left|\beta_{f}-\beta_{f, h}\right|\right) \\
& \quad+\left(\left\|\mathbf{u}_{p}-\mathbf{u}_{p, h}\right\|_{\mathbf{X}_{p}}+\left\|p_{p}-p_{p, h}\right\|_{L^{2}\left(\Omega_{p}\right)}+\left|\beta_{p}-\beta_{p, h}\right|\right) \\
& \leq C \inf _{\widehat{p}_{I, h} \in L_{h}}\left\|p_{I}-\widehat{p}_{I, h}\right\|_{H^{\frac{1}{2}\left(\Gamma_{I}\right)}}+C\left(\inf _{\widehat{\mathbf{u}}_{h} \in \mathbf{X}_{f, h}}\left\|\mathbf{u}_{f}-\widehat{\mathbf{u}}_{h}\right\|_{\mathbf{X}_{f}}+\inf _{\widehat{p}_{h} \in M_{f, h}}\left\|p_{f}-\widehat{p}_{h}\right\|_{L^{2}\left(\Omega_{f}\right)}\right) \\
& \quad+C\left(\inf _{\widehat{\mathbf{u}}_{h} \in \mathbf{X}_{p, h}}\left\|\mathbf{u}_{p}-\widehat{\mathbf{u}}_{h}\right\|_{\mathbf{X}_{p}}+\inf _{\widehat{p}_{h} \in M_{p, h}}\left\|p_{p}-\widehat{p}_{h}\right\|_{L^{2}\left(\Omega_{p}\right)}\right) .
\end{aligned}
$$

Proof. Let $\widehat{p}_{I, h} \in L_{h}$. From the weak formulation (3.48) and its finite dimensional counterpart (4.78), we have

$$
\begin{aligned}
A\left(p_{I}, p_{I, h}-\widehat{p}_{I, h}\right) & =0, \\
A_{h}\left(p_{I, h}, p_{I, h}-\widehat{p}_{I, h}\right) & =0 .
\end{aligned}
$$

These two orthogonality conditions together with the strict monotonicity of $A(\cdot, \cdot)$ yield

$$
\begin{aligned}
\frac{1}{C}\left\|p_{I, h}-\widehat{p}_{I, h}\right\|_{H^{\frac{1}{2}\left(\Gamma_{I}\right)}}^{2} \leq & A\left(p_{I, h}, p_{I, h}-\widehat{p}_{I, h}\right)-A\left(\widehat{p}_{I, h}, p_{I, h}-\widehat{p}_{I, h}\right) \\
= & A\left(p_{I, h}, p_{I, h}-\widehat{p}_{I, h}\right)-A_{h}\left(p_{I, h}, p_{I, h}-\widehat{p}_{I, h}\right) \\
& +A\left(p_{I}, p_{I, h}-\widehat{p}_{I, h}\right)-A\left(\widehat{p}_{I, h}, p_{I, h}-\widehat{p}_{I, h}\right) .
\end{aligned}
$$


The first two terms on the right-hand side of (4.81) contain the error propagated from the two subdomains and can be bounded using Theorems 12 and 13:

$$
\begin{aligned}
& A\left(p_{I, h}, p_{I, h}-\widehat{p}_{I, h}\right)-A_{h}\left(p_{I, h}, p_{I, h}-\widehat{p}_{I, h}\right) \\
& =-\left\langle\left(p_{I, h}-\widehat{p}_{I, h}\right),\left(\mathbf{u}_{f}^{*}\left(p_{I, h}\right)-\mathbf{u}_{f, h}^{*}\left(p_{I, h}\right)\right) \cdot \mathbf{n}_{f}\right\rangle_{\Gamma_{I}}-\left\langle\left(\mathbf{u}_{p}^{*}\left(p_{I, h}\right)-\mathbf{u}_{p, h}^{*}\left(p_{I, h}\right)\right) \cdot \mathbf{n}_{p},\left(p_{I, h}-\widehat{p}_{I, h}\right)\right\rangle_{\Gamma_{I}} \\
& \leq C\left\|\mathbf{u}_{f}^{*}\left(p_{I, h}\right)-\mathbf{u}_{f, h}^{*}\left(p_{I, h}\right)\right\|_{\mathbf{X}_{f}}\left\|p_{I, h}-\widehat{p}_{I, h}\right\|_{H^{-\frac{1}{2}\left(\Gamma_{I}\right)}}+C\left\|\mathbf{u}_{p}^{*}\left(p_{I, h}\right)-\mathbf{u}_{p, h}^{*}\left(p_{I, h}\right)\right\|_{\mathbf{X}_{p}}\left\|p_{I, h}-\widehat{p}_{I, h}\right\|_{H^{\frac{1}{2}}\left(\Gamma_{I}\right)} \\
& \leq C\left(\inf _{\widehat{\mathbf{u}}_{h} \in \mathbf{X}_{f, h}}\left\|\mathbf{u}_{f}-\widehat{\mathbf{u}}_{h}\right\|_{\mathbf{X}_{f}}+\inf _{\widehat{p}_{h} \in M_{f, h}}\left\|p_{f}-\widehat{p}_{h}\right\|_{L^{2}\left(\Omega_{f}\right)}\right)\left\|p_{I, h}-\widehat{p}_{I, h}\right\|_{H^{-\frac{1}{2}}\left(\Gamma_{I}\right)} \\
& +C\left(\inf _{\widehat{\mathbf{u}}_{h} \in \mathbf{X}_{p, h}}\left\|\mathbf{u}_{p}-\widehat{\mathbf{u}}_{h}\right\|_{\mathbf{X}_{p}}+\inf _{\widehat{p}_{h} \in M_{p, h}}\left\|p_{p}-\widehat{p}_{h}\right\|_{L^{2}\left(\Omega_{p}\right)}\right)\left\|p_{I, h}-\widehat{p}_{I, h}\right\|_{H^{\frac{1}{2}}\left(\Gamma_{I}\right)} \\
& \leq \epsilon\left\|p_{I, h}-\widehat{p}_{I, h}\right\|_{H^{\frac{1}{2}}\left(\Gamma_{I}\right)}^{2}+C\left(\inf _{\widehat{\mathbf{u}}_{h} \in \mathbf{X}_{f, h}}\left\|\mathbf{u}_{f}-\widehat{\mathbf{u}}_{h}\right\|_{\mathbf{X}_{f}}^{2}+\inf _{\widehat{p}_{h} \in M_{f, h}}\left\|p_{f}-\widehat{p}_{h}\right\|_{L^{2}\left(\Omega_{f}\right)}^{2}\right) \\
& +C\left(\inf _{\widehat{\mathbf{u}}_{h} \in \mathbf{X}_{p, h}}\left\|\mathbf{u}_{p}-\widehat{\mathbf{u}}_{h}\right\|_{\mathbf{X}_{p}}^{2}+\inf _{\hat{p}_{h} \in M_{p, h}}\left\|p_{p}-\widehat{p}_{h}\right\|_{L^{2}\left(\Omega_{p}\right)}^{2}\right) .
\end{aligned}
$$

The last two terms in (4.81) contain the error from the interface discretization:

$$
\begin{aligned}
& A\left(p_{I}, p_{I, h}-\widehat{p}_{I, h}\right)-A\left(\widehat{p}_{I, h}, p_{I, h}-\widehat{p}_{I, h}\right) \\
& =-\left\langle\left(p_{I, h}-\widehat{p}_{I, h}\right),\left(\mathbf{u}_{f}^{*}\left(p_{I}\right)-\mathbf{u}_{f}^{*}\left(p_{I, h}\right)\right) \cdot \mathbf{n}_{f}\right\rangle_{\Gamma_{I}}-\left\langle\left(\mathbf{u}_{p}^{*}\left(p_{I}\right)-\mathbf{u}_{p}^{*}\left(p_{I, h}\right)\right) \cdot \mathbf{n}_{p},\left(p_{I, h}-\widehat{p}_{I, h}\right)\right\rangle_{\Gamma_{I}} \\
& \leq C\left\|\mathbf{u}_{f}^{*}\left(p_{I}\right)-\mathbf{u}_{f}^{*}\left(\widehat{p}_{I, h}\right)\right\|_{\mathbf{X}_{f}}\left\|p_{I, h}-\widehat{p}_{I, h}\right\|_{H^{-\frac{1}{2}\left(\Gamma_{I}\right)}}+C\left\|\mathbf{u}_{p}^{*}\left(p_{I}\right)-\mathbf{u}_{p}^{*}\left(\widehat{p}_{I, h}\right)\right\|_{\mathbf{X}_{p}}\left\|p_{I, h}-\widehat{p}_{I, h}\right\|_{H^{\frac{1}{2}}\left(\Gamma_{I}\right)} \\
& \leq C\left\|p_{I}-\widehat{p}_{I, h}\right\|_{H^{-\frac{1}{2}\left(\Gamma_{I}\right)}}\left\|p_{I, h}-\widehat{p}_{I, h}\right\|_{H^{-\frac{1}{2}\left(\Gamma_{I}\right)}}+C\left\|p_{I}-\widehat{p}_{I, h}\right\|_{H^{\frac{1}{2}}\left(\Gamma_{I}\right)}\left\|p_{I, h}-\widehat{p}_{I, h}\right\|_{H^{\frac{1}{2}}\left(\Gamma_{I}\right)} \\
& \leq C\left\|p_{I}-\widehat{p}_{I, h}\right\|_{H^{\frac{1}{2}\left(\Gamma_{I}\right)}}^{2}+\epsilon\left\|p_{I, h}-\widehat{p}_{I, h}\right\|_{H^{\frac{1}{2}}\left(\Gamma_{I}\right)}^{2} .
\end{aligned}
$$

This theorem follows by first noting the fact that $\widehat{p}_{I, h}$ can be chosen arbitrarily from $L_{h}$ and then applying a triangle inequality and Theorems 12 and 13.

\section{$5 \quad$ Numerical Examples}

We consider a coupled Stokes-Darcy system on the domain $[0,2] \times[0,1]$, representing a twodimensional version of an industrial filtering application where a non-Newtonian fluid passes through a filter to remove unwanted particulates. Here the flow of the fluid through the channel $[0,1] \times[0,1]$ is coupled with its flow in the porous medium $[1,2] \times[0,1]$. We impose a defective boundary condition on the left boundary by specifying an inflow flux of one, and we impose a defective boundary condition on the right boundary of an outflow flux of one. Along the top and bottom boundaries, we impose a no-slip boundary condition for the Stokes flow and a no-flow boundary condition for the Darcy flow. We assume a Cross model for the fluid viscosity $\nu_{f}$ in the Stokes region and another Cross model for the effective viscosity $\nu_{p}$ in the Darcy region:

$$
\begin{gathered}
\nu_{f}(|D \mathbf{u}|)=\nu_{f, \infty}+\frac{\nu_{f, 0}-\nu_{f, \infty}}{1+K_{f}|D \mathbf{u}|^{2-r_{f}}}, \\
\nu_{p}(|\mathbf{u}|)=\nu_{p, \infty}+\frac{\nu_{p, 0}-\nu_{p, \infty}}{1+K_{p}|\mathbf{u}|^{2-r_{p}}},
\end{gathered}
$$


where $|\cdot|$ is the Euclidean norm in $\mathbb{R}^{d}$ or the Frobenius norm in $\mathbb{R}^{d \times d}$. In our numerical examples below, we set $K_{f}=K_{p}=1, \nu_{f, \infty}=\nu_{p, \infty}=1, \nu_{f, 0}=\nu_{p, 0}=10, r_{f}=r_{p}=1$. We note that a fluid with $r_{f}, r_{p} \in[1,2)$ possesses a shear thinning property, and $r_{f}=r_{p}=2$ corresponds to the special case of a Newtonian fluid. On the interface $\{1\} \times[0,1]$ of the two subdomains, we impose the Beavers-Joseph-Saffman slip condition with $\alpha_{S}=1$, in addition to the conservation of mass and normal forces across this interface. The permeability of the porous medium is set to be the identity matrix in $\mathbb{R}^{2 \times 2}$.

For the numerical discretization, we use a $20 \times 10$ uniform rectangular mesh. The Taylor-Hood space of $Q_{2}-Q_{1}$ is used for the Stokes flow; that is, velocity is approximated by a continuous piecewise biquadratic polynomial and pressure is approximated by a continuous piecewise bilinear polynomial. The Darcy flow is approximated by the $\mathrm{RT}_{1}$ space (i.e. velocity is approximated by a piecewise biquadratic polynomial with certain normal-component continuities and pressure is approximated by a piecewise bilinear polynomial). The mortar finite element space on the interface is the continuous linear polynomial space. Since the pressure is unique only up to an additive constant in this problem, we impose one additional constraint to enforce a zero average of the pressure on the outflow boundary $\{2\} \times[0,1]$. (We have also ran numerical simulations on a mesh containing 400 triangles, using $P_{2}-P_{1}$ Taylor-Hood for Stokes, $\mathrm{RT}_{1}$ for Darcy, and continuous $P_{1}$ for the mortar space on $\Gamma_{I}$. The results with triangular meshes (not shown) are almost identical to the results presented here.)

The approximating system of coupled equations has two features to note. Firstly, the equations are non-linear and secondly, the coupled system can be recast as an interface problem. Depending upon the order in which these features are implemented gives rise to two approximating algorithms.

\section{Algorithm 1}

- In the outer loop solve the (non-linear) interface problem.

- In the inner loop solve a (decoupled) non-linear problem on each subdomain.

\section{Algorithm 2}

- In the outer loop solve the (coupled) non-linear problem.

- In the inner loop solve the linear interface problem.

We plan to investigate the performance of the two algorithms in a forthcoming paper. For the computations presented herein we use a modification of Algorithm 2. As the total degrees of freedom is small, for the inner loop we simply use a sparse direct solver.

Figure 5.1 displays the simulation results obtained using the defective boundary condition treatment described in this paper. (See the Remark following Theorem 1.) Physically this boundary condition (BCI) corresponds to an inflow that is connected to a large reservior of water. Note that in Figure 5.1 that the fluid velocity near the inflow corners is not parallel to the top and bottom boundaries.

We have also considered the physical circumstance where the inflow boundary is attached to another same-sized channel supplying the fluid. For this case (BCII) we supplement the specified flow rate constraint with the condition that, at the inflow, the tangential component of the velocity is zero. Equation (3.16) is replaced by

$$
\int_{\Gamma_{f, F}}\left(\boldsymbol{\sigma}_{i j} n_{j}-p n_{j}\right) v_{i} d s=\int_{\Gamma_{f, F}} \beta_{f} v_{i} n_{i} d s+\sum_{m=1}^{d-1} \int_{\Gamma_{f, F}} \chi_{f, m} v_{i} t_{m, i} d s .
$$


Equation (3.17) remains unchanged, and we add the constraint:

$$
\sum_{m=1}^{d-1} \int_{\Gamma_{f, F}} \psi_{f, m} u_{i} t_{m, i} d s=0 .
$$

Here the Lagrange multiplier $\beta_{f} \in \mathbb{R}^{K_{f}}=\mathbb{R}$ represents the normal component of the traction vector (a constant function on $\left.\Gamma_{f, F}=\{0\} \times[0,1]\right)$. The function $\chi_{f} \in\left(H^{-\frac{1}{2}}\left(\Gamma_{f, F}\right)\right)^{d-1}=H^{-\frac{1}{2}}\left(\Gamma_{f, F}\right)$ (for $d=2$ ) is an arbitrary function so that the tangential component of the traction vector is unconstrained; but the function $\psi_{f} \in\left(H^{-\frac{1}{2}}\left(\Gamma_{f, F}\right)\right)^{d-1}=H^{-\frac{1}{2}}\left(\Gamma_{f, F}\right)$ serves to weakly enforce a zero tangential component of the velocity. In the finite element approximation, we discretize $H^{-\frac{1}{2}}\left(\Gamma_{f, F}\right)$ by a continuous piecewise quadratic polynomial function.

Results using BCII are presented in Figure 5.2. Note that at the inflow the veolicty is parallel to the top and bottom boundaries.
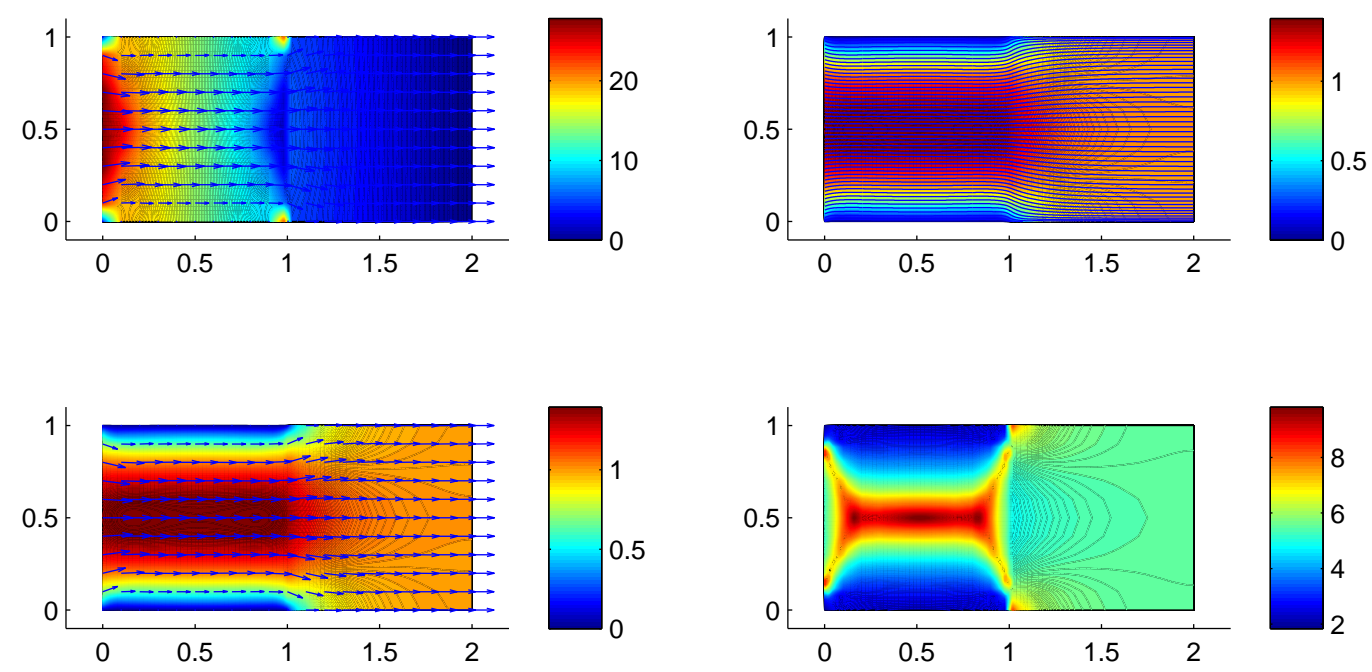

Figure 5.1: Polymeric fluid flowing from a channel into a porous medium: simulation results using the defective boundary condition treatment I. Top left: pressure contour together with velocity arrows; Top right: velocity magnitude contour with streamlines; Bottom left: velocity magnitude contour with velocity arrows; Bottom right: viscosity contour.

\section{Conclusions}

We have analyzed a multiphysics coupling strategy for nonlinear Stokes and Darcy flows which allows for separate resolution of the flows in each domain. The strategy is easily parallelized and enables use of existing Stokes and Darcy flow codes. 

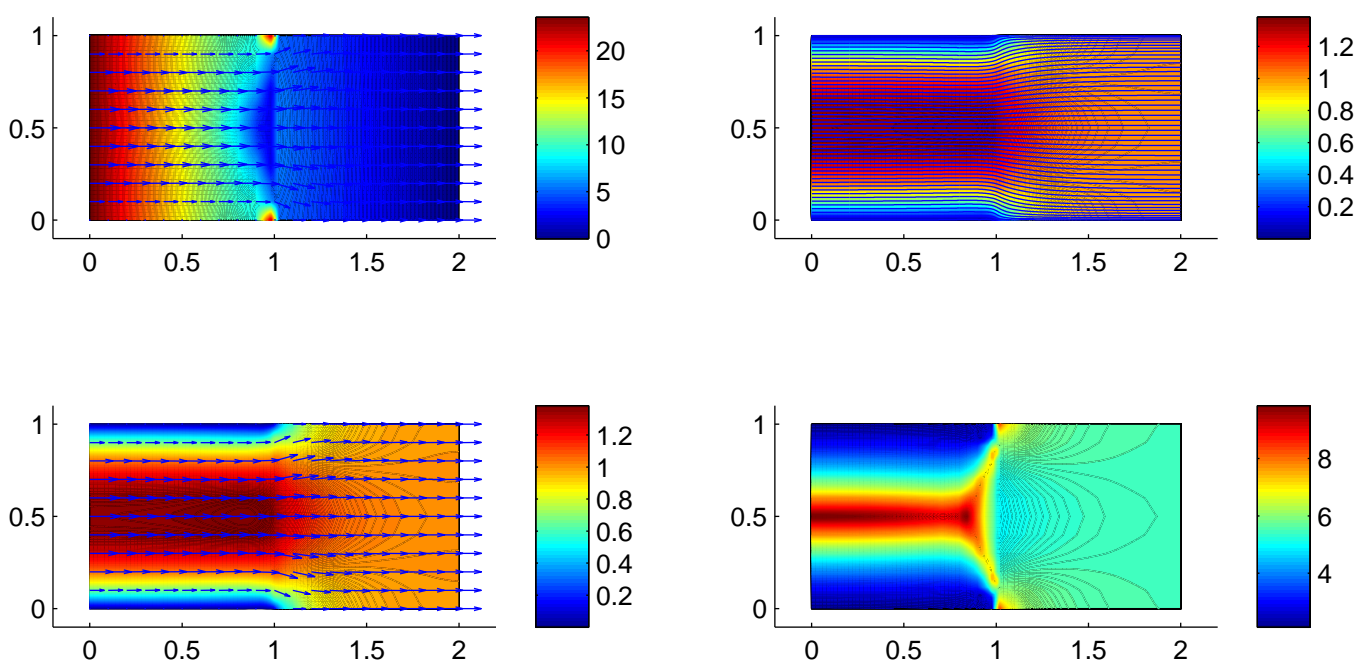

Figure 5.2: Polymeric fluid flowing from a channel into a porous medium: simulation results using the defective boundary condition treatment II. Top left: pressure contour together with velocity arrows; Top right: velocity magnitude contour with streamlines; Bottom left: velocity magnitude contour with velocity arrows; Bottom right: viscosity contour.

The domains are coupled through conservation of mass and balance of normal forces along the interface, along with a condition on the tangential component of stress in the Stokes region. This has been the standard practice for coupling of Newtonian fluids, and we have extended these results to handle the non-Newtonian case where viscosities are velocity dependent. We have shown existence and uniqueness of the variational solution and have presented numerical results to demonstrate the method.

We can see the effects of the no-slip and zero transmissibility boundary conditions in the Stokes and Darcy regions, respectively. The viscosity is relatively large around the central axis of the Stokes region due to the small strain rate and decreases as the strain rate increases towards the boundary. In comparison, the viscosity in the Darcy region has much less variation due to the (almost) uniform velocity profile.

We have also presented two realizations of the defective boundary conditions that match the physical scenarios we wish to model. The first treatment (BCI) corresponds to an inflow boundary that is connected to a large reservoir of fluid, while the second treatment (BCII) corresponds to a case where the inflow boundary is attached to another same-sized channel supplying the fluid. The results show the differences in the pressure, velocity, and viscosity profiles for each of these situations. As expected, the velocity streamlines and viscosity contours show the viscosity effects at the inflow for BCI. The pressure and velocity of the fluid are clearly affected by the change in momentum between $\mathrm{BCI}$ and BCII.

These results, combined with earlier analyses of coupled Stokes-Darcy problems, will allow for 
simulation of variety of filtration processes. Future work will incorporate coupled transport and reaction equations across the flow domain to more completely capture problems of interest.

\section{Appendix}

\section{A On Inf-Sup Conditions}

Theorem 18. Let $V, Q_{1}, Q_{2}$ be Hilbert spaces; $b_{1}(\cdot, \cdot): V \times Q_{1} \longrightarrow \mathbb{R}, b_{2}(\cdot, \cdot): V \times Q_{2} \longrightarrow \mathbb{R}$ be continuous bilinear functionals; and $Z_{1}:=\left\{v \in V \mid b_{1}(v, q)=0, \forall q \in Q_{1}\right\}$ be a null space. Let $C_{k}$, $k=1,2,12$, be positive constants. Then the inf-sup conditions (A.1) and (A.2) are equivalent to the combined inf-sup condition (A.3).

$$
\begin{gathered}
\sup _{v \in V,\|v\|_{V}=1} b_{1}\left(v, q_{1}\right) \geq C_{1}\left\|q_{1}\right\|_{Q_{1}}, \quad \forall q_{1} \in Q_{1}, \\
\sup _{v \in Z_{1},\|v\|_{V}=1} b_{2}\left(v, q_{2}\right) \geq C_{2}\left\|q_{2}\right\|_{Q_{2}}, \quad \forall q_{2} \in Q_{2}, \\
\sup _{v \in V,\|v\|_{V=1}}\left(b_{1}\left(v, q_{1}\right)+b_{2}\left(v, q_{2}\right)\right) \geq C_{12}\left(\left\|q_{1}\right\|_{Q_{1}}+\left\|q_{2}\right\|_{Q_{2}}\right), \quad \forall\left(q_{1}, q_{2}\right) \in Q_{1} \times Q_{2} .
\end{gathered}
$$

Remark. This theorem can be extended to the case of multiple bilinear functionals $b_{k}(\cdot, \cdot): V \times$ $Q_{k} \longrightarrow \mathbb{R}, k=1,2,3, \cdots K$.

Proof. The implication of (A.3) from (A.1) and (A.2) was been shown in [11]. It is trivial to show that (A.3) implies (A.1). We now assume that (A.3) and (A.1) hold, and we show (A.2) below.

Without loss of generality, we assume that $Z_{1}$ is a proper subspace of $V$; that is, $Z_{1}^{\perp}$ is a nontrivial space that contains nonzero functions. Given a function $v \in V$, we let $P_{Z_{1}} v$ be the orthogonal projection of $v$ onto $Z_{1}$ and we define $P_{Z_{1}^{\perp}} v:=v-P_{Z_{1}} v \in Z_{1}^{\perp}$. We remark that $P_{Z_{1}}$ is well defined as $Z_{1}$ is a closed subspace of the Hilbert space $V$. In addition, we know that there exists a constant $C_{Z_{1}}=1$ such that

$$
\left\|P_{Z_{1}} v\right\|_{V} \leq C_{Z_{1}}\|v\|_{V}
$$

Given a function $q_{2} \in Q_{2}$, we look for a solution $q_{1} \in Q_{1}$ such that

$$
b_{1}\left(v_{1}, q_{1}\right)=-b_{2}\left(v_{1}, q_{2}\right), \quad \forall v_{1} \in Z_{1}^{\perp} .
$$

We denote the solution $q_{1}$ by $\Pi_{Q_{1}} q_{2}$. We note that $b_{1}(\cdot, \cdot)$ is a continuous bilinear form; the righthand side in (A.4) is a continuous linear functional for a fixed $q_{2} ; b_{1}(\cdot, \cdot)$ satisfies the inf-sup condition (A.1) and $\sup _{q_{1} \in Q_{1}} b_{1}\left(v_{1}, q_{1}\right)>0$ for any nonzero element $v_{1} \in Z_{1}^{\perp}$. Consequently, the generalized Lax-Milgram theorem is applicable, which implies that the solution to (A.4) indeed exists and is unique. 
The properties of $P_{Z_{1}}$ and $\Pi_{Q_{1}}$, together with (A.3), yield the desired result:

$$
\begin{aligned}
\sup _{v_{1} \in Z_{1},\left\|v_{1}\right\|_{V}=1} b_{2}\left(v_{1}, q_{2}\right) & \geq \sup _{v \in V,\left\|P_{Z_{1}} v\right\|_{V}=1} b_{2}\left(P_{Z_{1}} v, q_{2}\right) \\
& =\frac{\|v\|_{V}}{\left\|P_{Z_{1}} v\right\|_{V}} \sup _{v \in V,\|v\|_{V}=1} b_{2}\left(P_{Z_{1}} v, q_{2}\right) \\
& \geq \frac{1}{C_{Z_{1}}} \sup _{v \in V,\|v\|_{V}=1} b_{2}\left(P_{Z_{1}} v, q_{2}\right) \\
& =\frac{1}{C_{Z_{1}}} \sup _{v \in V,\|v\|_{V}=1}\left(b_{1}\left(P_{Z_{1}} v, \Pi_{Q_{1}} q_{2}\right)+b_{2}\left(P_{Z_{1}} v, q_{2}\right)\right) \\
& =\frac{1}{C_{Z_{1}}} \sup _{v \in V,\|v\|_{V}=1}\left(b_{1}\left(P_{Z_{1}} v+P_{Z_{1}^{\perp}} v, \Pi_{Q_{1}} q_{2}\right)+b_{2}\left(P_{Z_{1}} v+P_{Z_{1}^{\perp}} v, q_{2}\right)\right) \\
& =\frac{1}{C_{Z_{1}}} \sup _{v \in V,\|v\|_{V}=1}\left(b_{1}\left(v, \Pi_{Q_{1}} q_{2}\right)+b_{2}\left(v, q_{2}\right)\right) \\
& \geq \frac{C_{12}}{C_{Z_{1}}}\left(\left\|\Pi_{Q_{1}} q_{2}\right\|_{Q_{1}}+\left\|q_{2}\right\|_{Q_{2}}\right) \\
& \geq \frac{C_{12}}{C_{Z_{1}}}\left\|q_{2}\right\|_{Q_{2}} .
\end{aligned}
$$

Remark. The equivalence of the combined inf-sup condition to the individual inf-sup conditions shown in Theorem 18 is similar to recent work of Gatica and Sayas [15].

\section{References}

[1] A. Bourgeat, O. Gipouloux, and E. Marusić-Paloka. Mathematical modelling and numerical simulation of a non-Newtonian viscous flow through a thin filter. SIAM J. Appl. Math., 62(2):597-626, 2001.

[2] S.C. Brenner and L.R. Scott. The Mathematical Theory of Finite Element Methods. Springer, New York, 2007.

[3] N. Bugli. How does air filter performance affect engine life? American Filtration and Separations Society, December 2008. One minute filtration article.

[4] E. Burman and P. Hansbo. A unified stabilized method for Stokes' and Darcy's equations. J. Comput. Appl. Math., 198:35-51, 2007.

[5] C.L. Cox, E.W. Jenkins, and P.J. Mucha. Modeling of debris deposition in a polymer extrusion filter. In PPS-21, Leipzig, Germany, June 2005.

[6] M. Discacciati, E. Miglio, and A. Quateroni. Mathematical and numerical models for coupling surface and groundwater flows. Appl. Numer. Math., 43:57-74, 2002.

[7] M. Discacciati and A. Quateroni. Analysis of a domain decomposition method for the coupling of Stokes and Darcy equations. In F. Brezzi, Buffa A, S. Corsaro, and A. Murli, editors, Numer. Math. Adv. Appl. - Enumath 2001, pages 3-20, Milan, 2003. Springer-Verlag. 
[8] M. Discacciati and A. Quateroni. Convergence analysis of a subdomain iterative method for the finite element approximation of the coupling of Stokes and Darcy equations. Comput. Vis. Sci., 6:93-103, 2004.

[9] M. Discacciati, A. Quateroni, and A. Valli. Robin-Robin domain decomposition methods for the Stokes-Darcy coupling. SIAM J. Numer. Anal., 45:1246-1268, 2007.

[10] D.D. Edie and C.H. Gooding. Prediction of pressure drop for the flow of polymer melts through sintered metal filters. Industrial and Engineering Chemistry Process Design and Development, 24:8-12, 1985.

[11] V.J. Ervin, E.W. Jenkins, and S. Sun. Coupled generalized nonlinear Stokes flow with flow through a porous medium. SIAM J. Numer. Anal., 47:929-952, 2009.

[12] J.P. Fues. High-efficiency filtration as pretreatment to membrane-based demineralization systems. In Proceedings of the American Filtration and Separations Society Annual Conference, May 2008.

[13] J. Galvis and M. Sarkis. BDD and FETI methods for mortar coupling of Stokes-Darcy systems. Serie A 563, Instituto de Matematica Pura e Aplicada, Brazil, January 2007.

[14] J. Galvis and M. Sarkis. Non-matching mortar discretization analysis for the coupling StokesDarcy equations. Electron. Trans. Numer. Anal., 26:350-384, 2007.

[15] G.N. Gatica and F.-J. Sayas. Characterizing the inf-sup condition on product spaces. Numer. Math., 109:209-231, 2008.

[16] V. Girault, S. Sun, M. F. Wheeler, and I. Yotov. Coupling discontinuous Galerkin and mixed finite element discretizations using mortar finite elements. SIAM J. Numer. Anal., 46(2):949979, 2008.

[17] R. Glowinski and M. F. Wheeler. Domain decomposition and mixed finite element methods for elliptic problems. In R. Glowinski, G. H. Golub, G. A. Meurant, and J. Periaux, editors, First International Symposium on Domain Decomposition Methods for Partial Differential Equations, pages 144-172, Philadelphia, 1988. SIAM.

[18] N.S. Hanspal, A.N. Waghode, V. Nassehi, and R.J. Wakeman. Numerical analysis of coupled Stokes/Darcy flow in industrial filtrations. Transp. Porous Media, 64:73-101, 2006.

[19] R.H.W. Hoppe, P. Porta, and Y. Vassilevski. Computational issues related to interative coupling of subsurface and channel flows. Calcolo, 44:1-20, 2007.

[20] K. Keller, T. Friedman, and A. Boxman. The bioseparation needs for tomorrow. Trends in Biotechnology, 19(11), 2001.

[21] W. Kozicki. Viscoelastic flow in packed beds or porous media. The Canadian Journal of Chemical Engineering, 79, Feb. 2001.

[22] W.J. Layton, F. Schieweck, and I. Yotov. Coupling fluid flow with porous media flow. SIAM J. Numer. Anal., 40:2195-2218, 2003. 
[23] R. Villarroel López, S. Elmaleh, and N. Ghaffor. Cross-flow ultrafiltration of hydrocarbon emulsions. Journal of Membrane Science, 102:55-64, June 1995.

[24] M. Mu and J. Xu. A two-grid method of a mixed Stokes-Darcy model for coupling fluid flow with porous media flow. SIAM J. Numer. Anal., 45:1801-1813, 2007.

[25] J.R.A. Pearson and P.M.J. Tardy. Models for flow of non-Newtonian and complex fluids through porous media. J. Non-Newtonian Fluid Mech., 102:447-473, 2002.

[26] D.Y.H. Pui, C. Qi, N. Stanley, G. Oberdörster, and A. Maynard. Recirculating air filtration significantly reduces exposure to airborne nanoparticles. Environmental Health Perspectives, 116(7), July 2008.

[27] B. Riviére. Analysis of a discontinuous finite element method for the coupled Stokes and Darcy problems. J. Sci. Comput., 22/23:479-500, 2005.

[28] B. Riviére and I. Yotov. Locally conservative coupling of Stokes and Darcy flows. SIAM J. Numer. Anal., 42:1959-1977, 2005.

[29] B. Seyfzadeh, D.A. Zumbrunnen, and R.A. Ross. Non-Newtonian flow and debris deposition in an extrusion filter medium. In Plastics - The Lone Star, pages 340-344. Society of Plastics Engineers, 2001.

[30] Y.-S. Wu and K. Pruess. A numerical method for simulating non-Newtonian fluid flow and displacement in porous media. Adv. Water Resour., 21(5):351-362, 1998. 